Research Article

\title{
Simpson's Integral Inequalities for Twice Differentiable Convex Functions
}

\author{
Miguel Vivas-Cortez $(1),{ }^{1}$ Thabet Abdeljawad $(1),{ }^{2,3,4}$ Pshtiwan Othman Mohammed, ${ }^{5}$ \\ and Yenny Rangel-Oliveros ${ }^{1}$ \\ ${ }^{1}$ Facultad de Ciencias Exactas y Naturales, Escuela de Ciencias Fisicas y Matematica, \\ Pontificia Universidad Católica Del Ecuador, Quito, Ecuador \\ ${ }^{2}$ Department of Mathematics and General Sciences, Prince Sultan University, P.O. Box 66833, Riyadh 11586, Saudi Arabia \\ ${ }^{3}$ Department of Medical Research, China Medical University, Taichung 40402, Taiwan \\ ${ }^{4}$ Department of Computer Science and Information Engineering, Asia University, Taichung, Taiwan \\ ${ }^{5}$ Department of Mathematics, College of Education, University of Sulaimani, Sulaimani, Kurdistan Region, Iraq
}

Correspondence should be addressed to Thabet Abdeljawad; tabdeljawad@psu.edu.sa

Received 30 March 2020; Accepted 25 May 2020; Published 27 June 2020

Guest Editor: Praveen Agarwal

Copyright (c) 2020 Miguel Vivas-Cortez et al. This is an open access article distributed under the Creative Commons Attribution License, which permits unrestricted use, distribution, and reproduction in any medium, provided the original work is properly cited.

Integral inequality is an interesting mathematical model due to its wide and significant applications in mathematical analysis and fractional calculus. In the present research article, we obtain new inequalities of Simpson's integral type based on the $\varphi$-convex and $\varphi$-quasiconvex functions in the second derivative sense. In the last sections, some applications on special functions are provided and shown via two figures to demonstrate the explanation of the readers.

\section{Introduction}

Integral inequality is a modern model of approximation theory that describes the growth rate of competing mathematical analysis. This model is also used in various fields such as ordinary differential equations $[1-5]$ and fractional calculus [6-17].

Among the several known inequalities, the most simple is Simpson's type, which has been successfully applied in several models of ordinary differential equations [18-29] and fractional differential equations [30-32]. Simpson's integral inequality is as follows: for any four times continuously differentiable function $\bar{F}:\left[\xi_{1}, \xi_{2}\right] \longrightarrow R$ on $\left(\xi_{1}, \xi_{2}\right)$, Simpson's integral inequality is defined as follows:

$$
\begin{aligned}
\mid & \frac{1}{3}\left[\frac{\bar{F}\left(\xi_{1}\right)+\bar{F}\left(\xi_{1}\right)}{2}+2 \bar{F}\left(\frac{\xi_{1}+\xi_{2}}{2}\right)\right] \\
& -\frac{1}{\xi_{2}-\xi_{1}} \int_{\xi_{1}}^{\xi_{2}} \bar{F}(x) \mathrm{d} x \mid \leq \frac{1}{2880}\left\|\bar{F}^{(4)}\right\|_{\infty}\left(\xi_{2}-\xi_{1}\right)^{4},
\end{aligned}
$$

where $\left\|\bar{F}^{(4)}\right\|_{\infty}=\sup _{x \in\left(\xi_{1}, \xi_{2}\right)}\left|\bar{F}^{(4)}(x)\right|<\infty$.

If the function $\bar{F}$ is neither four times differentiable nor is the fourth derivative $\bar{F}^{(4)}$ bounded on $\left(\xi_{1}, \xi_{2}\right)$, then we cannot apply the classical Simpson quadrature formula.

The following literature results obtained by Alomari et al. [18] and Sarikaya et al. [23] become a special case in our findings in Sections 2 and 3.

Lemma 1 (see [18]). Let $\bar{F}: \mathfrak{J} \longrightarrow R$ be twice differentiable function on $\mathfrak{I}$ with $\bar{F}^{\prime \prime} \in L_{1}\left[\xi_{1}, \xi_{2}\right]$, then we have

$$
\begin{aligned}
& \frac{\bar{F}\left(\xi_{1}\right)+\bar{F}\left(\xi_{2}\right)}{2}-\frac{1}{\xi_{2}-\xi_{1}} \int_{\xi_{1}}^{\xi_{2}} \bar{F}(x) \mathrm{d} x \\
& =\frac{\left(\xi_{2}-\xi_{1}\right)^{2}}{2} \int_{0}^{1} t(1-t) \bar{F}^{\prime \prime}\left(t \xi_{1}+(1-t) \xi_{2}\right) \mathrm{d} t .
\end{aligned}
$$

Lemma 2 (see [23]). Let $\bar{F}: \mathfrak{I} \longrightarrow R$ be twice differentiable function on $\mathfrak{I}$ such that $\bar{F}^{\prime \prime} \in L_{1}\left[\xi_{1}, \xi_{2}\right]$, where $\xi_{1}, \xi_{2} \in \mathfrak{F}$ with $\xi_{1}<\xi_{2}$, then we have 


$$
\begin{gathered}
\frac{1}{6}\left[\bar{F}\left(\xi_{1}\right)+4 \bar{F}\left(\frac{\xi_{1}+\xi_{2}}{2}\right)+\bar{F}\left(\xi_{2}\right)\right]-\frac{1}{\xi_{2}-\xi_{1}} \int_{\xi_{1}}^{\xi_{2}} \bar{F}(x) \mathrm{d} x \\
=\left(\xi_{2}-\xi_{1}\right)^{2} \int_{0}^{1} \hbar(t) \bar{F}^{\prime \prime}\left(t \xi_{2}+(1-t) \xi_{1}\right) \mathrm{d} t,
\end{gathered}
$$

where

$$
\hbar(t)= \begin{cases}\frac{t}{2}\left(\frac{1}{3}-t\right) ; & \text { if } t \in\left[0, \frac{1}{2}\right), \\ (1-t)\left(\frac{t}{2}-\frac{1}{3}\right) ; & \text { if } t \in\left[\frac{1}{2}, 1\right] .\end{cases}
$$

Through this paper, $R$ represents the set of real numbers and $\mathfrak{J}$ be an interval in $R$ and $\varphi: R \times R \longrightarrow R$ be a bifunction apart from some special cases.

This paper deals with the notations of $\varphi$-convex and $\varphi$-quasiconvex functions which were introduced by Gordji et al. [33] as follows.

Definition 1. A function $\bar{F}: \mathfrak{J} \longrightarrow R$ is called convex with respect to $\varphi$ (or briefly $\varphi$-convex), if

$$
\bar{F}\left(t \xi_{1}+(1-t) \xi_{2}\right) \leq \bar{F}\left(\xi_{2}\right)+t \varphi\left(\bar{F}\left(\xi_{1}\right), \bar{F}\left(\xi_{2}\right)\right),
$$

for all $\xi_{1}, \xi_{2} \in \mathfrak{J}$ and $t \in[0,1]$. Furthermore, $\bar{F}$ is called $\varphi$-quasiconvex, if

$$
\bar{F}\left(t \xi_{1}+(1-t) \xi_{2}\right) \leq \max \left\{\bar{F}\left(\xi_{2}\right), \bar{F}\left(\xi_{2}\right)+\varphi\left(\bar{F}\left(\xi_{1}\right), \bar{F}\left(\xi_{2}\right)\right)\right\},
$$

for all $\xi_{1}, \xi_{2} \in \mathfrak{J}$ and $t \in[0,1]$.

Remark 1. (i) It is easy to see the definition that every $\varphi$-convex function is $\varphi$-quasiconvex

(ii) If we take $\varphi\left(\xi_{1}, \xi_{2}\right)=\xi_{1}-\xi_{2}$ in Definition 1 , then the definitions of $\varphi$-convex and $\varphi$-quasiconvex are reduced to the definition of convex function and quasiconvex function, respectively

Next, we will give examples for the above definitions.

Example 1. Let $\bar{F}(x)=x^{2}$, then $\bar{F}$ is convex and $\varphi$-convex with $\varphi\left(\gamma_{1}, \gamma_{2}\right)=2 \gamma_{1}+\gamma_{2}$; indeed,

$$
\begin{aligned}
\bar{F}\left(t \xi_{1}+(1-t) \xi_{2}\right) & =\left(t \xi_{1}+(1-t) \xi_{2}\right)^{2} \\
& \leq \xi_{2}^{2}+t \xi_{1}^{2}+2 t(1-t) \xi_{1} \xi_{2} \\
& \leq \xi_{2}^{2}+t \xi_{1}^{2}+t(1-t)\left(\xi_{1}^{2}+\xi_{2}^{2}\right) \\
& \leq \xi_{2}^{2}+t\left(\xi_{1}^{2}+\xi_{1}^{2}+\xi_{2}^{2}\right) \\
& =\bar{F}\left(\xi_{2}\right)+t \varphi\left(\bar{F}\left(\xi_{1}\right), \bar{F}\left(\xi_{2}\right)\right) .
\end{aligned}
$$

Example 2. Let $\bar{F}(x)=x^{3}$, then $\bar{F}$ is not convex but is $\varphi$-convex with $\varphi\left(\gamma_{1}, \gamma_{2}\right)=3 \gamma_{2}^{2}\left(\gamma_{1}-\gamma_{2}\right)+3 \gamma_{2}\left(\gamma_{1}-\gamma_{2}\right)^{2}+$ $\left(\gamma_{1}-\gamma_{2}\right)^{3}$; indeed,

$$
\begin{aligned}
\bar{F}\left(t \xi_{1}+(1-t) \xi_{2}\right) & =\left(t \xi_{1}+(1-t) \xi_{2}\right)^{3}=\left(\xi_{2}+t\left(\xi_{1}-\xi_{2}\right)\right)^{3} \\
& =\xi_{2}^{3}+3 \xi_{2}^{2} t\left(\xi_{1}-\xi_{2}\right)+3 \xi_{2} t^{2}\left(\xi_{1}-\xi_{2}\right)^{2}+t^{3}\left(\xi_{1}-\xi_{2}\right)^{3} \\
& =\bar{F}\left(\xi_{2}\right)+t\left[3 \xi_{2}^{2}\left(\xi_{1}-\xi_{2}\right)+3 \xi_{2} t\left(\xi_{1}-\xi_{2}\right)^{2}+t^{2}\left(\xi_{1}-\xi_{2}\right)^{3}\right] \\
& \leq \bar{F}\left(\xi_{2}\right)+t\left[3 \xi_{2}^{2}\left(\xi_{1}-\xi_{2}\right)+3 \xi_{2}\left(\xi_{1}-\xi_{2}\right)^{2}+\left(\xi_{1}-\xi_{2}\right)^{3}\right] \\
& =\bar{F}\left(\xi_{2}\right)+t \varphi\left(\bar{F}\left(\xi_{1}\right), \bar{F}\left(\xi_{2}\right)\right) .
\end{aligned}
$$

Example 3. Let $\bar{F}:\left[\xi_{1}, \xi_{2}\right] \longrightarrow R, 0<\xi_{1}<\xi_{2}$, with $\bar{F}(x)=1 / x^{2}$. We observe that $\bar{F}$ is convex on $\left[\xi_{1}, \xi_{2}\right]$ and therefore $\varphi$-quasiconvex with $\varphi\left(\gamma_{1}, \gamma_{2}\right)=\gamma_{1}-\gamma_{2}$.

Example 4. Let $\bar{F}:\left[\xi_{1}, \xi_{2}\right] \longrightarrow R, 0<\xi_{1}<\xi_{2}$, with $\bar{F}(x)=2 / x^{3}$. We observe that $\bar{F}$ is convex on $\left[\xi_{1}, \xi_{2}\right]$ and therefore $\varphi$-quasiconvex with $\varphi\left(\gamma_{1}, \gamma_{2}\right)=\gamma_{1}-\gamma_{2}$.

Example 5. Let $\bar{F}:\left[\xi_{1}, \xi_{2}\right] \longrightarrow R, 0<\xi_{1}<\xi_{2}$, with $\bar{F}(x)=2$. We obviously see that $\bar{F}$ is $\varphi$-quasiconvex with $\varphi\left(\gamma_{1}, \gamma_{2}\right)=\gamma_{1}-\gamma_{2}$.

The essential object of this study is to establish new Simpson's integral inequalities for the $\varphi$-convex and $\varphi$-quasiconvex functions in the second derivative sense at certain powers.

\section{Simpson's Inequality for $\varphi$-Convex}

In this section, we give a new refinement of Simpson integral inequality for twice differentiable functions.

Theorem 1. Let $\bar{F}: \mathfrak{I} \longrightarrow R$ be a twice differentiable function on $\mathfrak{J}$ such that $\bar{F}^{\prime \prime} \in L_{1}\left[\xi_{1}, \xi_{2}\right]$, where $\xi_{1}, \xi_{2} \in \mathfrak{J}$ with $\xi_{1}<\xi_{2}$. If $\left|\bar{F}^{\prime \prime}\right|$ is $\varphi$-convex on $\left[\xi_{1}, \xi_{2}\right]$, then we have

$$
\begin{aligned}
& \left|\frac{1}{6}\left[\bar{F}\left(\xi_{1}\right)+4 \bar{F}\left(\frac{\xi_{1}+\xi_{2}}{2}\right)+\bar{F}\left(\xi_{2}\right)\right]-\frac{1}{\xi_{2}-\xi_{1}} \int_{\xi_{1}}^{\xi_{2}} \bar{F}(x) \mathrm{d} x\right| \\
& \quad \leq \frac{\left(\xi_{2}-\xi_{1}\right)^{2}}{81}\left[\left|\bar{F}^{\prime \prime}\left(\xi_{1}\right)\right|+\frac{1}{2} \varphi\left(\left|\bar{F}^{\prime \prime}\left(\xi_{1}\right)\right|,\left|\bar{F}^{\prime \prime}\left(\xi_{2}\right)\right|\right)\right] .
\end{aligned}
$$


Proof. By making the use of Lemma 2 and the $\varphi$-convexity of $\left|\bar{F}^{\prime \prime}\right|$, we find that

$$
\begin{aligned}
& \left|\frac{1}{6}\left[\bar{F}\left(\xi_{1}\right)+4 \bar{F}\left(\frac{\xi_{1}+\xi_{2}}{2}\right)+\bar{F}\left(\xi_{2}\right)\right]-\frac{1}{\xi_{2}-\xi_{1}} \int_{\xi_{1}}^{\xi_{2}} \bar{F}(x) \mathrm{d} x\right| \\
& \quad \leq\left(\xi_{2}-\xi_{1}\right)^{2} \int_{0}^{1}|k(t)|\left|\bar{F}^{\prime \prime}\left(t \xi_{2}+(1-t) \xi_{1}\right)\right| \mathrm{d} t \\
& \leq\left(\xi_{2}-\xi_{1}\right)^{2} \int_{0}^{1 / 2}\left|\frac{t}{2}\left(\frac{1}{3}-t\right)\right|\left(\left|\bar{F}^{\prime \prime}\left(\xi_{1}\right)\right|+t \varphi\left(\left|\bar{F}^{\prime \prime}\left(\xi_{1}\right)\right|,\left|\bar{F}^{\prime \prime}\left(\xi_{2}\right)\right|\right)\right) \mathrm{d} t \\
& \quad+\left(\xi_{2}-\xi_{1}\right)^{2} \int_{1 / 2}^{1}\left|(1-t)\left(\frac{t}{2}-\frac{1}{3}\right)\right|\left(\left|\bar{F}^{\prime \prime}\left(\xi_{1}\right)\right|+t \varphi\left(\left|\bar{F}^{\prime \prime}\left(\xi_{1}\right)\right|,\left|\bar{F}^{\prime \prime}\left(\xi_{2}\right)\right|\right)\right) \mathrm{d} t \\
& \quad:=q\left(\xi_{2}-\xi_{1}\right)^{2}\left(\top_{1}+\mathrm{T}_{2}\right),
\end{aligned}
$$

where

$$
\begin{aligned}
& \top_{1}:=\int_{0}^{1 / 2}\left|\frac{t}{2}\left(\frac{1}{3}-t\right)\right|\left(\left|\bar{F}^{\prime \prime}\left(\xi_{1}\right)\right|+t \varphi\left(\left|\bar{F}^{\prime \prime}\left(\xi_{1}\right)\right|,\left|\bar{F}^{\prime \prime}\left(\xi_{2}\right)\right|\right)\right) \mathrm{d} t \\
& =\int_{0}^{1 / 2}\left|\frac{t}{2}\left(\frac{1}{3}-t\right)\right|\left|\bar{F}^{\prime \prime}\left(\xi_{1}\right)\right| \mathrm{d} t+\int_{0}^{1 / 2}\left|\frac{t}{2}\left(\frac{1}{3}-t\right)\right| t \varphi\left(\left|\bar{F}^{\prime \prime}\left(\xi_{1}\right)\right|,\left|\bar{F}^{\prime \prime}\left(\xi_{2}\right)\right|\right) \mathrm{d} t \\
& =\left|\bar{F}^{\prime \prime}\left(\xi_{1}\right)\right| \int_{0}^{\frac{1}{2}}\left|\frac{t}{2}\left(\frac{1}{3}-t\right)\right| \mathrm{d} t+\varphi\left(\left|\bar{F}^{\prime \prime}\left(\xi_{1}\right)\right|,\left|\bar{F}^{\prime \prime}\left(\xi_{2}\right)\right|\right) \int_{0}^{1 / 2}\left|\frac{t}{2}\left(\frac{1}{3}-t\right)\right| t \mathrm{~d} t \\
& =\left|\bar{F}^{\prime \prime}\left(\xi_{1}\right)\right| \int_{0}^{1 / 3} \frac{t}{2}\left(\frac{1}{3}-t\right) \mathrm{d} t-\left|\bar{F}^{\prime \prime}\left(\xi_{1}\right)\right| \int_{\frac{1}{3}}^{1 / 2} \frac{t}{2}\left(\frac{1}{3}-t\right) \mathrm{d} t \\
& +\varphi\left(\left|\bar{F}^{\prime \prime}\left(\xi_{1}\right)\right|,\left|\bar{F}^{\prime \prime}\left(\xi_{2}\right)\right|\right) \int_{0}^{1 / 3} \frac{t}{2}\left(\frac{1}{3}-t\right) t \mathrm{~d} t-\varphi\left(\left|\bar{F}^{\prime \prime}\left(\xi_{1}\right)\right|,\left|\bar{F}^{\prime \prime}\left(\xi_{2}\right)\right|\right) \int_{1 / 3}^{1 / 2} \frac{t}{2}\left(\frac{1}{3}-t\right) t \mathrm{~d} t \\
& =\frac{1}{162}\left|\bar{F}^{\prime \prime}\left(\xi_{1}\right)\right|+\frac{59}{31104} \varphi\left(\left|\bar{F}^{\prime \prime}\left(\xi_{1}\right)\right|,\left|\bar{F}^{\prime \prime}\left(\xi_{2}\right)\right|\right) \\
& \top_{2}:=\int_{1 / 2}^{1}\left|(1-t)\left(\frac{t}{2}-\frac{1}{3}\right)\right|\left(\left|\bar{F}^{\prime \prime}\left(\xi_{1}\right)\right|+t \varphi\left(\left|\bar{F}^{\prime \prime}\left(\xi_{1}\right)\right|,\left|\bar{F}^{\prime \prime}\left(\xi_{2}\right)\right|\right)\right) \mathrm{d} t \\
& =\int_{1 / 2}^{1}\left|(1-t)\left(\frac{t}{2}-\frac{1}{3}\right)\right|\left|\bar{F}^{\prime \prime}\left(\xi_{1}\right)\right| \mathrm{d} t+\int_{1 / 2}^{1}\left|(1-t)\left(\frac{t}{2}-\frac{1}{3}\right)\right| t \varphi\left(\left|\bar{F}^{\prime \prime}\left(\xi_{1}\right)\right|,\left|\bar{F}^{\prime \prime}\left(\xi_{2}\right)\right|\right) \mathrm{d} t \\
& =\left|\bar{F}^{\prime \prime}\left(\xi_{1}\right)\right| \int_{1 / 2}^{1}\left|(1-t)\left(\frac{t}{2}-\frac{1}{3}\right)\right| \mathrm{d} t+\varphi\left(\left|\bar{F}^{\prime \prime}\left(\xi_{1}\right)\right|,\left|\bar{F}^{\prime \prime}\left(\xi_{2}\right)\right|\right) \int_{1 / 2}^{1}\left|(1-t)\left(\frac{t}{2}-\frac{1}{3}\right)\right| t \mathrm{~d} t \\
& =\frac{1}{162}\left|\bar{F}^{\prime \prime}\left(\xi_{1}\right)\right|+\frac{133}{31104} \varphi\left(\left|\bar{F}^{\prime \prime}\left(\xi_{1}\right)\right|,\left|\bar{F}^{\prime \prime}\left(\xi_{2}\right)\right|\right)
\end{aligned}
$$


Corollary 1. Theorem 1 with $\bar{F}\left(\xi_{1}\right)=\bar{F}\left(\left(\xi_{1}+\xi_{2}\right) / 2\right)=\bar{F}\left(\xi_{2}\right)$ gives the following new inequality:

$$
\begin{aligned}
& \left|\frac{1}{\xi_{2}-\xi_{1}} \int_{\xi_{1}}^{\xi_{2}} \bar{F}(x) d x-\bar{F}\left(\frac{\xi_{1}+\xi_{2}}{2}\right)\right| \\
& \quad \leq \frac{\left(\xi_{2}-\xi_{1}\right)^{2}}{81}\left[\left|\bar{F}^{\prime \prime}\left(\xi_{1}\right)\right|+\frac{1}{2} \varphi\left(\left|\bar{F}^{\prime \prime}\left(\xi_{1}\right)\right|,\left|\bar{F}^{\prime \prime}\left(\xi_{2}\right)\right|\right)\right] .
\end{aligned}
$$

Remark 2. Inequality (9) with $\varphi\left(\left|\bar{F}^{\prime \prime}\left(\xi_{1}\right)\right|,\left|\bar{F}^{\prime \prime}\left(\xi_{2}\right)\right|\right)=$ $\left|\bar{F}^{\prime \prime}\left(\xi_{2}\right)\right|-\left|\bar{F}^{\prime \prime}\left(\xi_{1}\right)\right|$ becomes

$$
\begin{aligned}
& \left|\frac{1}{6}\left[\bar{F}\left(\xi_{1}\right)+4 \bar{F}\left(\frac{\xi_{1}+\xi_{2}}{2}\right)+\bar{F}\left(\xi_{2}\right)\right]-\frac{1}{\xi_{2}-\xi_{1}} \int_{\xi_{1}}^{\xi_{2}} \bar{F}(x) \mathrm{d} x\right| \\
& \quad \leq \frac{\left(\xi_{2}-\xi_{1}\right)^{2}}{162}\left[\left|\bar{F}^{\prime \prime}\left(\xi_{1}\right)\right|+\left|\bar{F}^{\prime \prime}\left(\xi_{2}\right)\right|\right] .
\end{aligned}
$$

Moreover, inequality (12) with $\varphi\left(\left|\bar{F}^{\prime \prime}\left(\xi_{1}\right)\right|,\left|\bar{F}^{\prime \prime}\left(\xi_{2}\right)\right|\right)=$ $\left|\bar{F}^{\prime \prime}\left(\xi_{2}\right)\right|-\left|\bar{F}^{\prime \prime}\left(\xi_{1}\right)\right|$ becomes

$$
\begin{aligned}
& \left|\frac{1}{\xi_{2}-\xi_{1}} \int_{\xi_{1}}^{\xi_{2}} \bar{F}(x) \mathrm{d} x-\bar{F}\left(\frac{\xi_{1}+\xi_{2}}{2}\right)\right| \\
& \quad \leq \frac{\left(\xi_{2}-\xi_{1}\right)^{2}}{162}\left[\left|\bar{F}^{\prime \prime}\left(\xi_{1}\right)\right|+\left|\bar{F}^{\prime \prime}\left(\xi_{2}\right)\right|\right] .
\end{aligned}
$$

These are both obtained by Sarikaya et al. [23] in Theorem 2.2 and Corollary 2.3, respectively.

Theorem 2. Let $\bar{F}: \mathfrak{I} \longrightarrow R$ be a twice differentiable function on $\mathfrak{I}$ such that $\bar{F}^{\prime \prime} \in L_{1}\left[\xi_{1}, \xi_{2}\right]$, where $\xi_{1}, \xi_{2} \in \mathfrak{I}$ with $\xi_{1}<\xi_{2}$. If $\left|\bar{F}^{\prime \prime}\right|^{q}$ is $\varphi$-convex on $\left[\xi_{1}, \xi_{2}\right]$ and $q \geq 1$, then we have

$$
\begin{aligned}
\left|\frac{1}{6}\left[\bar{F}\left(\xi_{1}\right)+4 \bar{F}\left(\frac{\xi_{1}+\xi_{2}}{2}\right)+\bar{F}\left(\xi_{2}\right)\right]-\frac{1}{\xi_{2}-\xi_{1}} \int_{\xi_{1}}^{\xi_{2}} \bar{F}(x) \mathrm{d} x\right| \\
\leq\left(\xi_{2}-\xi_{1}\right)^{2}\left(\frac{1}{162}\right)^{1-(1 / q)}\left\{\left(\frac{1}{162}\left|\bar{F}^{\prime \prime}\left(\xi_{1}\right)\right|^{q}\right.\right. \\
\left.\quad+\frac{59}{31104} \varphi\left(\left|\bar{F}^{\prime \prime}\left(\xi_{1}\right)\right|^{q},\left|\bar{F}^{\prime \prime}\left(\xi_{2}\right)\right|^{q}\right)\right)^{1 / q} \\
\left.\quad+\left(\frac{1}{162}\left|\bar{F}^{\prime \prime}\left(\xi_{1}\right)\right|^{q}+\frac{133}{31104} \varphi\left(\left|\bar{F}^{\prime \prime}\left(\xi_{1}\right)\right|^{q},\left|\bar{F}^{\prime \prime}\left(\xi_{2}\right)\right|^{q}\right)\right)^{\frac{1}{q}}\right\},
\end{aligned}
$$

where $1 / p+1 / q=1$.

Proof. Let $q \geq 1$, then by using Lemma 2, we have

$$
\begin{aligned}
& \left|\frac{1}{6}\left[\bar{F}\left(\xi_{1}\right)+4 \bar{F}\left(\frac{\xi_{1}+\xi_{2}}{2}\right)+\bar{F}\left(\xi_{2}\right)\right]-\frac{1}{\xi_{2}-\xi_{1}} \int_{\xi_{1}}^{\xi_{2}} \bar{F}(x) \mathrm{d} x\right| \\
& \quad \leq\left(\xi_{2}-\xi_{1}\right)^{2} \int_{0}^{1}|k(t)|\left|\bar{F}^{\prime \prime}\left(t \xi_{2}+(1-t) \xi_{1}\right)\right| \mathrm{d} t \\
& \quad=\left(\xi_{2}-\xi_{1}\right)^{2} \int_{0}^{1 / 2}\left|\frac{t}{2}\left(\frac{1}{3}-t\right)\right|\left|\bar{F}^{\prime \prime}\left(t \xi_{2}+(1-t) \xi_{1}\right)\right| \mathrm{d} t \\
& \quad+\left(\xi_{2}-\xi_{1}\right)^{2} \int_{1 / 2}^{1}\left|(1-t)\left(\frac{t}{2}-\frac{1}{3}\right)\right|\left|\bar{F}^{\prime \prime}\left(t \xi_{2}+(1-t) \xi_{1}\right)\right| \mathrm{d} t .
\end{aligned}
$$

By making the use of the Hölder's inequality for the above integrals, we have

$$
\begin{aligned}
& \left|\frac{1}{6}\left[\bar{F}\left(\xi_{1}\right)+4 \bar{F}\left(\frac{\xi_{1}+\xi_{2}}{2}\right)+\bar{F}\left(\xi_{2}\right)\right]-\frac{1}{\xi_{2}-\xi_{1}} \int_{\xi_{1}}^{\xi_{2}} \bar{F}(x) \mathrm{d} x\right| \\
& \quad \leq\left(\xi_{2}-\xi_{1}\right)^{2}\left(\int_{0}^{1 / 2}\left|\frac{t}{2}\left(\frac{1}{3}-t\right)\right| \mathrm{d} t\right)^{1-1 / q}\left(\int_{0}^{1 / 2}\left|\frac{t}{2}\left(\frac{1}{3}-t\right)\right|\left|\bar{F}^{\prime \prime}\left(t \xi_{2}+(1-t) \xi_{1}\right)\right|^{q} \mathrm{~d} t\right)^{1 / q} \\
& \quad+\left(\xi_{2}-\xi_{1}\right)^{2}\left(\int_{1 / 2}^{1}\left|(1-t)\left(\frac{t}{2}-\frac{1}{3}\right)\right| \mathrm{d} t\right)^{1-1 / q}\left(\int_{1 / 2}^{1}\left|(1-t)\left(\frac{t}{2}-\frac{1}{3}\right)\right|\left|\bar{F}^{\prime \prime}\left(t \xi_{2}+(1-t) \xi_{1}\right)\right|^{q} \mathrm{~d} t\right)^{1 / q} .
\end{aligned}
$$

By $\varphi$-convexity of $\left|\bar{F}^{\prime \prime}\right|^{q}$ for the last two integrals, we have 


$$
\begin{aligned}
& \int_{0}^{1 / 2}\left|\frac{t}{2}\left(\frac{1}{3}-t\right)\right|\left|\bar{F}^{\prime \prime}\left(t \xi_{2}+(1-t) \xi_{1}\right)\right|^{q} \mathrm{~d} t \\
& \quad \leq \int_{0}^{1 / 2}\left|\frac{t}{2}\left(\frac{1}{3}-t\right)\right|\left[\left|\bar{F}^{\prime \prime}\left(\xi_{1}\right)\right|^{q}+t \varphi\left(\left|\bar{F}^{\prime \prime}\left(\xi_{1}\right)\right|^{q},\left|\bar{F}^{\prime \prime}\left(\xi_{2}\right)\right|^{q}\right)\right] \mathrm{d} t \\
& \quad=\left|\bar{F}^{\prime \prime}\left(\xi_{1}\right)\right|^{q} \int_{0}^{1 / 2}\left|\frac{t}{2}\left(\frac{1}{3}-t\right)\right| \mathrm{d} t+\varphi\left(\left|\bar{F}^{\prime \prime}\left(\xi_{1}\right)\right|^{q},\left|\bar{F}^{\prime \prime}\left(\xi_{2}\right)\right|^{q}\right) \int_{0}^{1 / 2}\left|\frac{t}{2}\left(\frac{1}{3}-t\right)\right| t \mathrm{~d} t \\
& \quad=\frac{1}{162}\left|\bar{F}^{\prime \prime}\left(\xi_{1}\right)\right|^{q}+\frac{59}{31104} \varphi\left(\left|\bar{F}^{\prime \prime}\left(\xi_{1}\right)\right|^{q},\left|\bar{F}^{\prime \prime}\left(\xi_{2}\right)\right|^{q}\right), \\
& \int_{1 / 2}^{1}\left|(1-t)\left(\frac{t}{2}-\frac{1}{3}\right)\right|\left|\bar{F}^{\prime \prime}\left(t \xi_{2}+(1-t) \xi_{1}\right)\right|^{q} \mathrm{~d} t \\
& \leq \int_{1 / 2}^{1}\left|(1-t)\left(\frac{t}{2}-\frac{1}{3}\right)\right|\left[\left|\bar{F}^{\prime \prime}\left(\xi_{1}\right)\right|^{q}+t \varphi\left(\left|\bar{F}^{\prime \prime}\left(\xi_{1}\right)\right|^{q},\left|\bar{F}^{\prime \prime}\left(\xi_{2}\right)\right|^{q}\right)\right] \mathrm{d} t \\
& \quad=\left|\bar{F}^{\prime \prime}\left(\xi_{1}\right)\right|^{q} \int_{\frac{1}{2}}^{1}\left|(1-t)\left(\frac{t}{2}-\frac{1}{3}\right)\right| \mathrm{d} t+\varphi\left(\left|\bar{F}^{\prime \prime}\left(\xi_{1}\right)\right|^{q},\left|\bar{F}^{\prime \prime}\left(\xi_{2}\right)\right|^{q}\right) \int_{1 / 2}^{1}\left|(1-t)\left(\frac{t}{2}-\frac{1}{3}\right)\right| t \mathrm{~d} t \\
& \quad=\frac{1}{162}\left|\bar{F}^{\prime \prime}\left(\xi_{1}\right)\right|^{q}+\frac{133}{31104} \varphi\left(\left|\bar{F}^{\prime \prime}\left(\xi_{1}\right)\right|^{q},\left|\bar{F}^{\prime \prime}\left(\xi_{2}\right)\right|^{q}\right) .
\end{aligned}
$$

By substituting (18) and (19) into (17), we have

$$
\begin{aligned}
\mid \frac{1}{6}[ & \left.\bar{F}\left(\xi_{1}\right)+4 \bar{F}\left(\frac{\xi_{1}+\xi_{2}}{2}\right)+\bar{F}\left(\xi_{2}\right)\right]-\frac{1}{\xi_{2}-\xi_{1}} \int_{\xi_{1}}^{\xi_{2}} \bar{F}(x) \mathrm{d} x \mid \\
\leq & \left(\xi_{2}-\xi_{1}\right)^{2}\left(\int_{0}^{1 / 2}\left|\frac{t}{2}\left(\frac{1}{3}-t\right)\right| \mathrm{d} t\right)^{1-1 / q}\left(\frac{1}{162}\left|\bar{F}^{\prime \prime}\left(\xi_{1}\right)\right|^{q}+\frac{59}{31104} \varphi\left(\left|\bar{F}^{\prime \prime}\left(\xi_{1}\right)\right|^{q},\left|\bar{F}^{\prime \prime}\left(\xi_{2}\right)\right|^{q}\right)\right)^{1 / q} \\
& +\left(\xi_{2}-\xi_{1}\right)^{2}\left(\int_{1 / 2}^{1}\left|(1-t)\left(\frac{t}{2}-\frac{1}{3}\right)\right| \mathrm{d} t\right)^{1-1 / q}\left(\frac{1}{162}\left|\bar{F}^{\prime \prime}\left(\xi_{1}\right)\right|^{q}+\frac{133}{31104} \varphi\left(\left|\bar{F}^{\prime \prime}\left(\xi_{1}\right)\right|^{q},\left|\bar{F}^{\prime \prime}\left(\xi_{2}\right)\right|^{q}\right)\right)^{1 / q} \\
= & \left(\xi_{2}-\xi_{1}\right)^{2}\left(\frac{1}{162}\right)^{1-1 / q}\left[\left(\frac{1}{162}\left|\bar{F}^{\prime \prime}\left(\xi_{1}\right)\right|^{q}+\frac{59}{31104} \varphi\left(\left|\bar{F}^{\prime \prime}\left(\xi_{1}\right)\right|^{q},\left|\bar{F}^{\prime \prime}\left(\xi_{2}\right)\right|^{q}\right)\right)^{1 / q}\right. \\
& \left.+\left(\frac{1}{162}\left|\bar{F}^{\prime \prime}\left(\xi_{1}\right)\right|^{q}+\frac{133}{31104} \varphi\left(\left|\bar{F}^{\prime \prime}\left(\xi_{1}\right)\right|^{q},\left|\bar{F}^{\prime \prime}\left(\xi_{2}\right)\right|^{q}\right)\right)^{1 / q}\right],
\end{aligned}
$$

where we used the identity

$$
\int_{0}^{1 / 2}\left|\frac{t}{2}\left(\frac{1}{3}-t\right)\right| \mathrm{d} t=\int_{1 / 2}^{1}\left|(1-t)\left(\frac{t}{2}-\frac{1}{3}\right)\right| \mathrm{d} t=\frac{1}{162} .
$$

Thus, we are done.
Corollary 2. Theorem 2 with $\bar{F}\left(\xi_{1}\right)=\bar{F}\left(\left(\xi_{1}+\xi_{2}\right) / 2\right)=\bar{F}\left(\xi_{2}\right)$ gives the following new inequality: 


$$
\begin{aligned}
& \left|\frac{1}{\xi_{2}-\xi_{1}} \int_{\xi_{1}}^{\xi_{2}} \bar{F}(x) \mathrm{d} x-\bar{F}\left(\frac{\xi_{1}+\xi_{2}}{2}\right)\right| \\
& \leq\left(\xi_{2}-\xi_{1}\right)^{2}\left(\frac{1}{162}\right)^{1-1 / q}\left[\left(\frac{1}{162}\left|\bar{F}^{\prime \prime}\left(\xi_{1}\right)\right|^{q}\right.\right. \\
& \left.\quad+\frac{59}{31104} \varphi\left(\left|\bar{F}^{\prime \prime}\left(\xi_{1}\right)\right|^{q},\left|\bar{F}^{\prime \prime}\left(\xi_{2}\right)\right|^{q}\right)\right)^{1 / q} \\
& \left.\quad+\left(\frac{1}{162}\left|\bar{F}^{\prime \prime}\left(\xi_{1}\right)\right|^{q}+\frac{133}{31104} \varphi\left(\left|\bar{F}^{\prime \prime}\left(\xi_{1}\right)\right|^{q},\left|\bar{F}^{\prime \prime}\left(\xi_{2}\right)\right|^{q}\right)\right)^{1 / q}\right] .
\end{aligned}
$$

Remark 3. Inequality (15) with $\varphi\left(\left|\bar{F}^{\prime \prime}\left(\xi_{1}\right)\right|^{q},\left|\bar{F}^{\prime \prime}\left(\xi_{2}\right)\right|^{q}\right)=$ $\left|\bar{F}^{\prime \prime}\left(\xi_{2}\right)\right|^{q}-\left|\bar{F}^{\prime \prime}\left(\xi_{1}\right)\right|^{q}$ becomes

$$
\begin{aligned}
& \left|\frac{1}{6}\left[\bar{F}\left(\xi_{1}\right)+4 \bar{F}\left(\frac{\xi_{1}+\xi_{2}}{2}\right)+\bar{F}\left(\xi_{2}\right)\right]-\frac{1}{\xi_{2}-\xi_{1}} \int_{\xi_{1}}^{\xi_{2}} \bar{F}(x) \mathrm{d} x\right| \\
& \leq\left(\xi_{2}-\xi_{1}\right)^{2}\left(\frac{1}{162}\right)^{1-1 / q}\left\{\left(\frac{133}{31104}\left|\bar{F}^{\prime \prime}\left(\xi_{1}\right)\right|^{q}+\frac{59}{31104}\left|\bar{F}^{\prime \prime}\left(\xi_{2}\right)\right|^{q}\right)^{1 / q}\right. \\
& \left.\quad+\left(\frac{59}{31104}\left|\bar{F}^{\prime \prime}\left(\xi_{1}\right)\right|^{q}+\frac{133}{31104}\left|\bar{F}^{\prime \prime}\left(\xi_{2}\right)\right|^{q}\right)^{1 / q}\right\} .
\end{aligned}
$$

Moreover, inequality (22) with $\varphi\left(\left|\bar{F}^{\prime \prime}\left(\xi_{1}\right)\right|^{q},\left|\bar{F}^{\prime \prime}\left(\xi_{2}\right)\right|^{q}\right)=$ $\left|\bar{F}^{\prime \prime}\left(\xi_{2}\right)\right|^{q}-\left|\bar{F}^{\prime \prime}\left(\xi_{1}\right)\right|^{q}$ becomes

$$
\begin{aligned}
& \left|\frac{1}{\xi_{2}-\xi_{1}} \int_{\xi_{1}}^{\xi_{2}} \bar{F}(x) \mathrm{d} x-\bar{F}\left(\frac{\xi_{1}+\xi_{2}}{2}\right)\right| \\
& \quad \leq\left(\xi_{2}-\xi_{1}\right)^{2}\left(\frac{1}{162}\right)^{1-1 / q}\left\{\left(\frac{133}{31104}\left|\bar{F}^{\prime \prime}\left(\xi_{1}\right)\right|^{q}+\frac{59}{31104}\left|\bar{F}^{\prime \prime}\left(\xi_{2}\right)\right|^{q}\right)^{1 / q}\right. \\
& \left.\quad+\left(\frac{59}{31104}\left|\bar{F}^{\prime \prime}\left(\xi_{1}\right)\right|^{q}+\frac{133}{31104}\left|\bar{F}^{\prime \prime}\left(\xi_{2}\right)\right|^{q}\right)^{1 / q}\right\} .
\end{aligned}
$$

These are both obtained by Sarikaya et al. [23] in Theorem 2.5 and Corollary 2.6, respectively.

Remark 4. Theorem 2 and Corollary 2 with $q=1$ become Theorem 1 and Corollary 1, respectively.

\section{Simpson's Inequality for $\varphi$-Quasiconvex}

Theorem 3. Let $\bar{F}: \mathfrak{I} \longrightarrow R$ be a twice differentiable function on $\mathfrak{\Im}$ provided $\bar{F}^{\prime \prime} \in L_{1}\left[\xi_{1}, \xi_{2}\right]$, where $\xi_{1}, \xi_{2} \in \mathfrak{\Im}$ with $\xi_{1}<\xi_{2}$. If $\left|\bar{F}^{\prime \prime}\right|$ is $\varphi$-quasiconvex on $\left[\xi_{1}, \xi_{2}\right]$, then we have

$$
\begin{aligned}
& \left|\frac{1}{6}\left[\bar{F}\left(\xi_{1}\right)+4 \bar{F}\left(\frac{\xi_{1}+\xi_{2}}{2}\right)+\bar{F}\left(\xi_{2}\right)\right]-\frac{1}{\xi_{2}-\xi_{1}} \int_{\xi_{1}}^{\xi_{2}} \bar{F}(x) \mathrm{d} x\right| \\
& \quad \leq \frac{\left(\xi_{2}-\xi_{1}\right)^{2}}{81} \max \left\{\left|\bar{F}^{\prime \prime}\left(\xi_{1}\right)\right|,\left|\bar{F}^{\prime \prime}\left(\xi_{1}\right)\right|+\varphi\left(\left|\bar{F}^{\prime \prime}\left(\xi_{2}\right)\right|,\left|\bar{F}^{\prime \prime}\left(\xi_{1}\right)\right|\right)\right\} .
\end{aligned}
$$

Proof. By making use of $\varphi$-quasiconvexity of $\left|\bar{F}^{\prime \prime}\right|$ and Lemma 2, we get

$$
\begin{aligned}
& \left|\frac{1}{6}\left[\bar{F}\left(\xi_{1}\right)+4 \bar{F}\left(\frac{\xi_{1}+\xi_{2}}{2}\right)+\bar{F}\left(\xi_{2}\right)\right]-\frac{1}{\xi_{2}-\xi_{1}} \int_{\xi_{1}}^{\xi_{2}} \bar{F}(x) \mathrm{d} x\right| \\
& \quad \leq\left(\xi_{2}-\xi_{1}\right)^{2} \int_{0}^{1}|k(t)|\left|\bar{F}^{\prime \prime}\left(t \xi_{2}+(1-t) \xi_{1}\right)\right| \mathrm{d} t \\
& \quad \leq\left(\xi_{2}-\xi_{1}\right)^{2} \int_{0}^{1 / 2}\left|\frac{t}{2}\left(\frac{1}{3}-t\right)\right| \max \left\{\left|\bar{F}^{\prime \prime}\left(\xi_{1}\right)\right|,\left|\bar{F}^{\prime \prime}\left(\xi_{1}\right)\right|+\varphi\left(\left|\bar{F}^{\prime \prime}\left(\xi_{2}\right)\right|,\left|\bar{F}^{\prime \prime}\left(\xi_{1}\right)\right|\right)\right\} \mathrm{d} t \\
& \quad+\left(\xi_{2}-\xi_{1}\right)^{2} \int_{1 / 2}^{1}\left|(1-t)\left(\frac{t}{2}-\frac{1}{3}\right)\right| \max \left\{\left|\bar{F}^{\prime \prime}\left(\xi_{1}\right)\right|,\left|\bar{F}^{\prime \prime}\left(\xi_{1}\right)\right|+\varphi\left(\left|\bar{F}^{\prime \prime}\left(\xi_{2}\right)\right|,\left|\bar{F}^{\prime \prime}\left(\xi_{1}\right)\right|\right)\right\} \mathrm{d} t \\
& =\left(\xi_{2}-\xi_{1}\right)^{2}\left(\overline{\mathrm{T}}_{1}+\overline{\mathrm{T}}_{2}\right),
\end{aligned}
$$


where

$$
\begin{aligned}
\overline{T_{1}} & =\int_{0}^{1 / 2}\left|\frac{t}{2}\left(\frac{1}{3}-t\right)\right| \max \left\{\left|\bar{F}^{\prime \prime}\left(\xi_{1}\right)\right|,\left|\bar{F}^{\prime \prime}\left(\xi_{1}\right)\right|+\varphi\left(\left|\bar{F}^{\prime \prime}\left(\xi_{2}\right)\right|,\left|\bar{F}^{\prime \prime}\left(\xi_{1}\right)\right|\right)\right\} \mathrm{d} t \\
& =\max \left\{\left|\bar{F}^{\prime \prime}\left(\xi_{1}\right)\right|,\left|\bar{F}^{\prime \prime}\left(\xi_{1}\right)\right|+\varphi\left(\left|\bar{F}^{\prime \prime}\left(\xi_{2}\right)\right|,\left|\bar{F}^{\prime \prime}\left(\xi_{1}\right)\right|\right)\right\} \int_{0}^{1 / 2}\left|\frac{t}{2}\left(\frac{1}{3}-t\right)\right| \mathrm{d} t \\
& =\max \left\{\left|\bar{F}^{\prime \prime}\left(\xi_{1}\right)\right|,\left|\bar{F}^{\prime \prime}\left(\xi_{1}\right)\right|+\varphi\left(\left|\bar{F}^{\prime \prime}\left(\xi_{2}\right)\right|,\left|\bar{F}^{\prime \prime}\left(\xi_{1}\right)\right|\right)\right\}\left[\int_{0}^{1 / 3} \frac{t}{2}\left(\frac{1}{3}-t\right) \mathrm{d} t-\int_{1 / 3}^{1 / 2} \frac{t}{2}\left(\frac{1}{3}-t\right) \mathrm{d} t\right] \\
& =\frac{1}{162} \max \left\{\left|\bar{F}^{\prime \prime}\left(\xi_{1}\right)\right|,\left|\bar{F}^{\prime \prime}\left(\xi_{1}\right)\right|+\varphi\left(\left|\bar{F}^{\prime \prime}\left(\xi_{2}\right)\right|,\left|\bar{F}^{\prime \prime}\left(\xi_{1}\right)\right|\right)\right\}, \\
\overline{T_{2}} & =\int_{1 / 2}^{1}\left|(1-t)\left(\frac{t}{2}-\frac{1}{3}\right)\right| \max \left\{\left|\bar{F}^{\prime \prime}\left(\xi_{1}\right)\right|,\left|\bar{F}^{\prime \prime}\left(\xi_{1}\right)\right|+\varphi\left(\left|\bar{F}^{\prime \prime}\left(\xi_{2}\right)\right|,\left|\bar{F}^{\prime \prime}\left(\xi_{1}\right)\right|\right)\right\} \mathrm{d} t \\
& =\max \left\{\left|\bar{F}^{\prime \prime}\left(\xi_{1}\right)\right|,\left|\bar{F}^{\prime \prime}\left(\xi_{1}\right)\right|+\varphi\left(\left|\bar{F}^{\prime \prime}\left(\xi_{2}\right)\right|,\left|\bar{F}^{\prime \prime}\left(\xi_{1}\right)\right|\right)\right\} \int_{1 / 2}^{1}\left|(1-t)\left(\frac{t}{2}-\frac{1}{3}\right)\right| \mathrm{d} t \\
& =\frac{1}{162} \max \left\{\left|\bar{F}^{\prime \prime}\left(\xi_{1}\right)\right|,\left|\bar{F}^{\prime \prime}\left(\xi_{1}\right)\right|+\varphi\left(\left|\bar{F}^{\prime \prime}\left(\xi_{2}\right)\right|,\left|\bar{F}^{\prime \prime}\left(\xi_{1}\right)\right|\right)\right\} .
\end{aligned}
$$

A simple rearrangement completes the proof.

Corollary 3. Theorem 3 with $\bar{F}\left(\xi_{1}\right)=\bar{F}\left(\left(\xi_{1}+\xi_{2}\right) / 2\right)=\bar{F}\left(\xi_{2}\right)$ becomes

$$
\left|\frac{1}{\xi_{2}-\xi_{1}} \int_{\xi_{1}}^{\xi_{2}} \bar{F}(x) \mathrm{d} x-\bar{F}\left(\frac{\xi_{1}+\xi_{2}}{2}\right)\right| \leq \frac{\left(\xi_{2}-\xi_{1}\right)^{2}}{81} \max \left\{\left|\bar{F}^{\prime \prime}\left(\xi_{1}\right)\right|,\left|\bar{F}^{\prime \prime}\left(\xi_{1}\right)\right|+\varphi\left(\left|\bar{F}^{\prime \prime}\left(\xi_{2}\right)\right|,\left|\bar{F}^{\prime \prime}\left(\xi_{1}\right)\right|\right)\right\} .
$$

Theorem 4. Let $\bar{F}: \mathfrak{I} \longrightarrow R$ be a twice differentiable function on $\mathfrak{J}$ provided $\bar{F}^{\prime \prime} \in L_{1}\left[\xi_{1}, \xi_{2}\right]$, where $\xi_{1}, \xi_{2} \in \mathfrak{I}$ with

$\xi_{1}<\xi_{2}$. If $\left|\bar{F}^{\prime \prime}\right|^{q}$ is $\varphi$-quasiconvex on $\left[\xi_{1}, \xi_{2}\right]$ and $q \geq 1$, then we have

$$
\begin{aligned}
& \left|\frac{1}{6}\left[\bar{F}\left(\xi_{1}\right)+4 \bar{F}\left(\frac{\xi_{1}+\xi_{2}}{2}\right)+\bar{F}\left(\xi_{2}\right)\right]-\frac{1}{\xi_{2}-\xi_{1}} \int_{\xi_{1}}^{\xi_{2}} \bar{F}(x) \mathrm{d} x\right| \\
& \quad \leq \frac{\left(\xi_{2}-\xi_{1}\right)^{2}}{81}\left(\max \left\{\left|\bar{F}^{\prime \prime}\left(\xi_{1}\right)\right|^{q},\left|\bar{F}^{\prime \prime}\left(\xi_{1}\right)\right|^{q}+\varphi\left(\left|\bar{F}^{\prime \prime}\left(\xi_{2}\right)\right|^{q},\left|\bar{F}^{\prime \prime}\left(\xi_{1}\right)\right|^{q}\right)\right\}\right)^{1 / q},
\end{aligned}
$$

where $1 / p+1 / q=1$.

Proof. Let $q \geq 1$, then by using Lemma 2, we have

$$
\begin{aligned}
\mid \frac{1}{6}[ & \left.\bar{F}\left(\xi_{1}\right)+4 \bar{F}\left(\frac{\xi_{1}+\xi_{2}}{2}\right)+\bar{F}\left(\xi_{2}\right)\right]-\frac{1}{\xi_{2}-\xi_{1}} \int_{\xi_{1}}^{\xi_{2}} \bar{F}(x) \mathrm{d} x \mid \\
\leq & \left(\xi_{2}-\xi_{1}\right)^{2} \int_{0}^{1}|k(t)|\left|\bar{F}^{\prime \prime}\left(t \xi_{2}+(1-t) \xi_{1}\right)\right| \mathrm{d} t \\
= & \left(\xi_{2}-\xi_{1}\right)^{2} \int_{0}^{1 / 2}\left|\frac{t}{2}\left(\frac{1}{3}-t\right)\right|\left|\bar{F}^{\prime \prime}\left(t \xi_{2}+(1-t) \xi_{1}\right)\right| \mathrm{d} t \\
& +\left(\xi_{2}-\xi_{1}\right)^{2} \int_{1 / 2}^{1}\left|(1-t)\left(\frac{t}{2}-\frac{1}{3}\right)\right|\left|\bar{F}^{\prime \prime}\left(t \xi_{2}+(1-t) \xi_{1}\right)\right| \mathrm{d} t .
\end{aligned}
$$


By making the use of the Hölder's inequality for the above integrals, we have

$$
\begin{aligned}
& \left|\frac{1}{6}\left[\bar{F}\left(\xi_{1}\right)+4 \bar{F}\left(\frac{\xi_{1}+\xi_{2}}{2}\right)+\bar{F}\left(\xi_{2}\right)\right]-\frac{1}{\xi_{2}-\xi_{1}} \int_{\xi_{1}}^{\xi_{2}} \bar{F}(x) \mathrm{d} x\right| \\
& \quad \leq\left(\xi_{2}-\xi_{1}\right)^{2}\left(\int_{0}^{1 / 2}\left|\frac{t}{2}\left(\frac{1}{3}-t\right)\right| \mathrm{d} t\right)^{1-(1 / q)}\left(\int_{0}^{1 / 2}\left|\frac{t}{2}\left(\frac{1}{3}-t\right)\right|\left|\bar{F}^{\prime \prime}\left(t \xi_{2}+(1-t) \xi_{1}\right)\right|^{q} \mathrm{~d} t\right)^{1 / q} \\
& \quad+\left(\xi_{2}-\xi_{1}\right)^{2}\left(\int_{\frac{1}{2}}^{1}\left|(1-t)\left(\frac{t}{2}-\frac{1}{3}\right)\right| \mathrm{d} t\right)^{1-(1 / q)}\left(\int_{1 / 2}^{1}\left|(1-t)\left(\frac{t}{2}-\frac{1}{3}\right)\right|\left|\bar{F}^{\prime \prime}\left(t \xi_{2}+(1-t) \xi_{1}\right)\right|^{q} \mathrm{~d} t\right)^{1 / q} .
\end{aligned}
$$
have

By $\varphi$-quasiconvexity of $\left|\bar{F}^{\prime \prime}\right|^{q}$ for the last two integrals, we

$$
\begin{aligned}
& \int_{0}^{1 / 2}\left|\frac{t}{2}\left(\frac{1}{3}-t\right)\right|\left|\bar{F}^{\prime \prime}\left(t \xi_{2}+(1-t) \xi_{1}\right)\right|^{q} \mathrm{~d} t \\
& \quad \leq \int_{0}^{1 / 2}\left|\frac{t}{2}\left(\frac{1}{3}-t\right)\right| \max \left\{\left|\bar{F}^{\prime \prime}\left(\xi_{1}\right)\right|^{q},\left|\bar{F}^{\prime \prime}\left(\xi_{1}\right)\right|^{q}+\varphi\left(\left|\bar{F}^{\prime \prime}\left(\xi_{2}\right)\right|^{q},\left|\bar{F}^{\prime \prime}\left(\xi_{1}\right)\right|^{q}\right)\right\} \mathrm{d} t \\
& \quad=\max \left\{\left|\bar{F}^{\prime \prime}\left(\xi_{1}\right)\right|^{q},\left|\bar{F}^{\prime \prime}\left(\xi_{1}\right)\right|^{q}+\varphi\left(\left|\bar{F}^{\prime \prime}\left(\xi_{2}\right)\right|^{q},\left|\bar{F}^{\prime \prime}\left(\xi_{1}\right)\right|^{q}\right)\right\} \int_{0}^{1 / 2}\left|\frac{t}{2}\left(\frac{1}{3}-t\right)\right| \mathrm{d} t \\
& \quad=\frac{1}{162} \max \left\{\left|\bar{F}^{\prime \prime}\left(\xi_{1}\right)\right|^{q},\left|\bar{F}^{\prime \prime}\left(\xi_{1}\right)\right|^{q}+\varphi\left(\left|\bar{F}^{\prime \prime}\left(\xi_{2}\right)\right|^{q},\left|\bar{F}^{\prime \prime}\left(\xi_{1}\right)\right|^{q}\right)\right\} \\
& \int_{1 / 2}^{1}\left|(1-t)\left(\frac{t}{2}-\frac{1}{3}\right)\right|\left|\bar{F}^{\prime \prime}\left(t \xi_{2}+(1-t) \xi_{1}\right)\right|^{q} \mathrm{~d} t \\
& \quad \leq \int_{1 / 2}^{1}\left|(1-t)\left(\frac{t}{2}-\frac{1}{3}\right)\right| \max \left\{\left|\bar{F}^{\prime \prime}\left(\xi_{1}\right)\right|^{q},\left|\bar{F}^{\prime \prime}\left(\xi_{1}\right)\right|^{q}+\varphi\left(\left|\bar{F}^{\prime \prime}\left(\xi_{2}\right)\right|^{q},\left|\bar{F}^{\prime \prime}\left(\xi_{1}\right)\right|^{q}\right)\right\} \mathrm{d} t \\
& \quad=\max \left\{\left|\bar{F}^{\prime \prime}\left(\xi_{1}\right)\right|^{q},\left|\bar{F}^{\prime \prime}\left(\xi_{1}\right)\right|^{q}+\varphi\left(\left|\bar{F}^{\prime \prime}\left(\xi_{2}\right)\right|^{q},\left|\bar{F}^{\prime \prime}\left(\xi_{1}\right)\right|^{q}\right)\right\} \int_{1 / 2}^{1}\left|(1-t)\left(\frac{t}{2}-\frac{1}{3}\right)\right| \mathrm{d} t \\
& \quad=\frac{1}{162} \max \left\{\left|\bar{F}^{\prime \prime}\left(\xi_{1}\right)\right|^{q},\left|\bar{F}^{\prime \prime}\left(\xi_{1}\right)\right|^{q}+\varphi\left(\left|\bar{F}^{\prime \prime}\left(\xi_{2}\right)\right|^{q},\left|\bar{F}^{\prime \prime}\left(\xi_{1}\right)\right|^{q}\right)\right\} .
\end{aligned}
$$

By substituting (32) and (33) into (31), we have

$$
\begin{aligned}
\mid \frac{1}{6}[ & \left.\bar{F}\left(\xi_{1}\right)+4 \bar{F}\left(\frac{\xi_{1}+\xi_{2}}{2}\right)+\bar{F}\left(\xi_{2}\right)\right]-\frac{1}{\xi_{2}-\xi_{1}} \int_{\xi_{1}}^{\xi_{2}} \bar{F}(x) \mathrm{d} x \mid \\
\leq & \left(\xi_{2}-\xi_{1}\right)^{2}\left(\int_{0}^{1 / 2}\left|\frac{t}{2}\left(\frac{1}{3}-t\right)\right| \mathrm{d} t\right)^{1-(1 / q)}\left(\frac{1}{162} \max \left\{\left|\bar{F}^{\prime \prime}\left(\xi_{1}\right)\right|^{q},\left|\bar{F}^{\prime \prime}\left(\xi_{1}\right)\right|^{q}+\varphi\left(\left|\bar{F}^{\prime \prime}\left(\xi_{2}\right)\right|^{q},\left|\bar{F}^{\prime \prime}\left(\xi_{1}\right)\right|^{q}\right)\right\}\right)^{1 / q} \\
& +\left(\xi_{2}-\xi_{1}\right)^{2}\left(\int_{1 / 2}^{1}\left|(1-t)\left(\frac{t}{2}-\frac{1}{3}\right)\right| \mathrm{d} t\right)^{1-(1 / q)}\left(\frac{1}{162} \max \left\{\left|\bar{F}^{\prime \prime}\left(\xi_{1}\right)\right|^{q},\left|\bar{F}^{\prime \prime}\left(\xi_{1}\right)\right|^{q}+\varphi\left(\left|\bar{F}^{\prime \prime}\left(\xi_{2}\right)\right|^{q},\left|\bar{F}^{\prime \prime}\left(\xi_{1}\right)\right|^{q}\right)\right\}\right)^{1 / q} \\
= & 2\left(\xi_{2}-\xi_{1}\right)^{2}\left(\frac{1}{162}\right)^{1-(1 / q)}\left(\frac{1}{162}\right)^{1 / q}\left(\max \left\{\left|\bar{F}^{\prime \prime}\left(\xi_{1}\right)\right|^{q},\left|\bar{F}^{\prime \prime}\left(\xi_{1}\right)\right|^{q}+\varphi\left(\left|\bar{F}^{\prime \prime}\left(\xi_{2}\right)\right|^{q},\left|\bar{F}^{\prime \prime}\left(\xi_{1}\right)\right|^{q}\right)\right\}\right)^{1 / q} \\
= & \frac{\left(\xi_{2}-\xi_{1}\right)^{2}}{81}\left(\max \left\{\left|\bar{F}^{\prime \prime}\left(\xi_{1}\right)\right|^{q},\left|\bar{F}^{\prime \prime}\left(\xi_{1}\right)\right|^{q}+\varphi\left(\left|\bar{F}^{\prime \prime}\left(\xi_{2}\right)\right|^{q},\left|\bar{F}^{\prime \prime}\left(\xi_{1}\right)\right|^{q}\right)\right\}\right)^{1 / q} .
\end{aligned}
$$


where we used the following identity

$$
\int_{0}^{1 / 2}\left|\frac{t}{2}\left(\frac{1}{3}-t\right)\right| \mathrm{d} t=\int_{1 / 2}^{1}\left|(1-t)\left(\frac{t}{2}-\frac{1}{3}\right)\right| \mathrm{d} t=\frac{1}{162} .
$$

Thus we are done.

Corollary 4. Theorem 4 with $\bar{F}\left(\xi_{1}\right)=\bar{F}\left(\left(\xi_{1}+\xi_{2}\right) / 2\right)=\bar{F}\left(\xi_{2}\right)$ becomes

$$
\begin{aligned}
& \left|\frac{1}{\xi_{2}-\xi_{1}} \int_{\xi_{1}}^{\xi_{2}} \bar{F}(x) \mathrm{d} x-\bar{F}\left(\frac{\xi_{1}+\xi_{2}}{2}\right)\right| \\
& \quad \leq \frac{\left(\xi_{2}-\xi_{1}\right)^{2}}{81}\left(\max \left\{\left|\bar{F}^{\prime \prime}\left(\xi_{1}\right)\right|^{q},\left|\bar{F}^{\prime \prime}\left(\xi_{1}\right)\right|^{q}+\varphi\left(\left|\bar{F}^{\prime \prime}\left(\xi_{2}\right)\right|^{q},\left|\bar{F}^{\prime \prime}\left(\xi_{1}\right)\right|^{q}\right)\right\}\right)^{1 / q} .
\end{aligned}
$$

Remark 5. Theorem 4 and Corollary 4 with $q=1$ become Theorem 3 and Corollary 3, respectively.
Corollary 5. Theorem 4 with $\bar{F}\left(\xi_{1}\right)=\bar{F}\left(\left(\xi_{1}+\xi_{2}\right) / 2\right)=\bar{F}\left(\xi_{2}\right)$ becomes

$$
\begin{aligned}
& \left|\frac{1}{\xi_{2}-\xi_{1}} \int_{\xi_{1}}^{\xi_{2}} \bar{F}(x) \mathrm{d} x-\bar{F}\left(\frac{\xi_{1}+\xi_{2}}{2}\right)\right| \\
& \quad \leq \frac{\left(\xi_{2}-\xi_{1}\right)^{2}}{81}\left(\max \left\{\left|\bar{F}^{\prime \prime}\left(\xi_{1}\right)\right|^{2},\left|\bar{F}^{\prime \prime}\left(\xi_{1}\right)\right|^{2}+\varphi\left(\left|\bar{F}^{\prime \prime}\left(\xi_{2}\right)\right|^{2},\left|\bar{F}^{\prime \prime}\left(\xi_{1}\right)\right|^{2}\right)\right\}\right)^{1 / 2} .
\end{aligned}
$$

\section{Applications}

Some applications for our findings are presented.

4.1. Applications to Special Means. The special means are itemized as follows:

(i) The arithmetic mean:

$$
\mathscr{A}=\mathscr{A}\left(\xi_{1}, \xi_{2}\right)=\frac{\xi_{1}+\xi_{2}}{2}, \quad \xi_{1}, \xi_{2} \geq 0 .
$$

(ii) The harmonic mean:

$$
\mathscr{H}=\mathscr{H}\left(\xi_{1}, \xi_{2}\right)=\frac{2 \xi_{1} \xi_{2}}{\xi_{1}+\xi_{2}}, \quad \xi_{1}, \xi_{2}>0 .
$$

(iii) The logarithmic mean:

$$
\mathscr{L}=\mathscr{L}\left(\xi_{1}, \xi_{2}\right)= \begin{cases}\frac{\xi_{2}-\xi_{1}}{\ln \xi_{2}-\ln \xi_{1}} ; & \text { if } \xi_{1} \neq \xi_{2}, \\ \xi_{1} ; & \text { if } \xi_{1}=\xi_{2},\end{cases}
$$

for $\xi_{1}, \xi_{2}>0$.

(iv) The $p$-logarithmic mean:

$$
\mathscr{L}_{p}=\mathscr{L}_{p}\left(\xi_{1}, \xi_{2}\right)= \begin{cases}{\left[\frac{\xi_{2}^{p+1}-\xi_{1}^{p+1}}{(p+1)\left(\xi_{2}-\xi_{1}\right)}\right]^{1 / p} ;} & \text { if } \xi_{1} \neq \xi_{2}, \\ \xi_{1} ; & \text { if } \xi_{1}=\xi_{2},\end{cases}
$$

for $p \in R \backslash\{-1,0\} ; \xi_{1}, \xi_{2}>0$.
We know that $\mathscr{L}_{p}$ is a monotonic nondecreasing function over $p \in R$ with $\mathscr{L}_{-1}=\mathscr{L}$. In particular, we can say that $\mathscr{H} \leq \mathscr{L} \leq \mathscr{A}$.

Now, using our findings in Section 2, we conclude the following new inequalities.

Proposition 1. Let $\xi_{1}, \xi_{2} \in R$ with $0<\xi_{1}<\xi_{2}$. Then, we have $\left|\frac{1}{3} \mathscr{A}\left(\xi_{1}^{4}, \xi_{2}^{4}\right)+\frac{2}{3} \mathscr{A}^{4}\left(\xi_{1}, \xi_{2}\right)-\mathscr{L}_{5}^{5}\left(\xi_{1}, \xi_{2}\right)\right| \leq \frac{\left(\xi_{2}-\xi_{1}\right)^{2}}{27}\left[8 \xi_{1}^{2}+\xi_{2}^{2}\right]$.

Proof. The assertion follows from Theorem 1 with $\bar{F}(x)=$ $x^{4} / 12, x \in\left[\xi_{1}, \xi_{2}\right]$ and a simple computation, where $\left|\bar{F}^{\prime \prime}\right|$ is $\varphi$-convex function with $\varphi(x, y)=2 x+y$ (see Example $1)$.

Proposition 2. Let $\xi_{1}, \xi_{2} \in R, 0<\xi_{1}<\xi_{2}$. Then, we have

$$
\begin{aligned}
\mid \frac{1}{3} & \mathscr{A}\left(\xi_{1}^{5}, \xi_{2}^{5}\right)+\frac{2}{3} \mathscr{A}^{5}\left(\xi_{1}, \xi_{2}\right)-\mathscr{L}_{6}^{6}\left(\xi_{1}, \xi_{2}\right) \mid \\
& \leq \frac{10\left(\xi_{2}-\xi_{1}\right)^{2}}{81}\left[2 \xi_{1}^{3}+\xi_{1}^{9}-\xi_{2}^{9}\right] .
\end{aligned}
$$

Proof. The assertion follows from Theorem 1 and a simple computation applied to $\bar{F}(x)=x^{5} / 20, x \in\left[\xi_{1}, \xi_{2}\right]$, where $\left|\bar{F}^{\prime \prime}\right|$ is $\varphi$-convex function with $\varphi(x, y)=3 y^{2}(x-y)+$ $3 y(x-y)^{2}+(x-y)^{3}$ (see Example 2).

The following proposition is a particular case of Corollary 11 in [34] when $\lambda=1 / 3$ (see Remark 12 in [34]). 
Proposition 3. Let $\xi_{1}, \xi_{2} \in R, 0<\xi_{1}<\xi_{2}$. Then, we have

$$
\left|\frac{1}{3} \mathscr{A}\left(\xi_{1}^{2}, \xi_{2}^{2}\right)+\frac{2}{3} \mathscr{A}^{2}\left(\xi_{1}, \xi_{2}\right)-\mathscr{L}_{3}^{3}\left(\xi_{1}, \xi_{2}\right)\right| \leq \frac{2\left(\xi_{2}-\xi_{1}\right)^{2}}{81} .
$$

Proof. The assertion follows from Theorem 3 and a simple computation applied to $\bar{F}(x)=x^{2}, x \in\left[\xi_{1}, \xi_{2}\right]$, where $\left|\bar{F}^{\prime \prime}(x)\right|=2$ is $\varphi$-quasiconvex function with $\varphi(x, y)=x-y$ (see Example 5).

Proposition 4. Let $\xi_{1}, \xi_{2} \in R, 0<\xi_{1}<\xi_{2}$. Then, for all $q>1$, we have

$$
\begin{aligned}
& \left|\frac{1}{3} \mathscr{H}^{-1}\left(\xi_{1}, \xi_{2}\right)+\frac{2}{3} \mathscr{A}^{-1}\left(\xi_{1}, \xi_{2}\right)-\mathscr{L}^{-1}\left(\xi_{1}, \xi_{2}\right)\right| \\
& \quad \leq \frac{\left(\xi_{2}-\xi_{1}\right)^{2}}{81} \max \left\{\frac{2^{q}}{\xi_{1}^{3 q}}, \frac{2^{q}}{\xi_{2}^{3 q}}\right\} .
\end{aligned}
$$

Proof. The assertion follows from Theorem 4 and a simple computation applied to $\bar{F}(x)=1 / x, x \in\left[\xi_{1}, \xi_{2}\right]$, where $\left|\bar{F}^{\prime \prime}(x)\right|=\left|2 / x^{3}\right|$ is $\varphi$-quasiconvex function with $\varphi(x, y)=$ $x-y$ (see Example 4).

4.2. Applications to Simpson's Formula. Let $\mathscr{P}$ be a partition of the interval $\left[\xi_{1}, \xi_{2}\right]$; that is $\mathscr{P}: \xi_{1}=s_{0}<s_{1}<\cdots<s_{n-1}$ $<s_{n}=\xi_{2} ; h_{i}=\left(s_{i+1}-s_{i}\right) / 2$ and consider Simpson's formula:

$$
S(\bar{F}, \mathscr{P})=\sum_{i=1}^{n-1} \frac{\bar{F}\left(s_{i}\right)+4 \bar{F}\left(s_{i}+h_{i}\right)+\bar{F}\left(s_{i+1}\right)}{6}\left(s_{i+1}-s_{i}\right) .
$$

We know that if $\bar{F}:\left[\xi_{1}, \xi_{2}\right] \longrightarrow R$ is differentiable such that $\bar{F}^{(q)}(x)$ exists on $\left(\xi_{1}, \xi_{2}\right)$ and $K=\max _{x \in\left[\xi_{1}, \xi_{2}\right]}\left|\bar{F}^{(q)}(x)\right|$ $<\infty$. Then, we have

$$
I=\int_{\xi_{1}}^{\xi_{2}} \bar{F}(s) \mathrm{d} s=S(\bar{F}, \mathscr{P})+E_{s}(\bar{F}, \mathscr{P})
$$

where the approximation error $E_{s}(\bar{F}, \mathrm{~L})$ satisfies

$$
\left|E_{s}(\bar{F}, \mathbf{L})\right| \leq \frac{K}{90} \sum_{i=0}^{n-1}\left(s_{i+1}-s_{i}\right)^{5} .
$$

It is clear that if the function $\bar{F}$ is not four times differentiable or $\bar{F}^{(4)}$ is not bounded on $\left(\xi_{1}, \xi_{2}\right)$, then (47) cannot be applied.

Theorem 5. Let $\bar{F}: \mathfrak{J} \subseteq[0, \infty) \longrightarrow R$ be a twice differentiable function on $\mathfrak{J}$ such that $\bar{F}^{\prime \prime} \in L_{1}\left[\xi_{1}, \xi_{2}\right]$, where $\xi_{1}, \xi_{2} \in \mathfrak{J}$ with $\xi_{1}<\xi_{2}$. If $\left|\bar{F}^{\prime \prime}\right|$ is $\varphi$-convex on $\left[\xi_{1}, \xi_{2}\right]$, then for every division $\mathscr{P}$ of $\left[\xi_{1}, \xi_{2}\right]$ we have

$$
\left|E_{s}(\bar{F}, \mathscr{P})\right| \leq \frac{1}{81} \sum_{i=0}^{n-1}\left(s_{i+1}-s_{i}\right)^{3}\left[\bar{F}^{\prime \prime}\left(s_{i}\right)+\frac{1}{2} \varphi\left(\bar{F}^{\prime \prime}\left(s_{i}\right), \bar{F}^{\prime \prime}\left(s_{i+1}\right)\right)\right] .
$$

Proof. By applying Theorem 1 on the subintervals $\left[s_{i}, s_{i+1}\right],(i=0,1,2, \ldots, n-1)$ of the division $\mathscr{P}$ to get

$$
\begin{aligned}
& \left|\frac{\left(s_{i+1}-s_{i}\right)}{6}\left[\bar{F}\left(s_{i}\right)+4 \bar{F}\left(\frac{s_{i+1}-s_{i}}{2}\right)+\bar{F}\left(s_{i+1}\right)\right]-\int_{s_{i}}^{s_{i+1}} \bar{F}(s) \mathrm{d} s\right| \\
& \leq \frac{\left(s_{i+1}-s_{i}\right)^{3}}{6}\left\{\bar{F}^{\prime \prime}\left(s_{i}\right)+\frac{1}{2} \varphi\left(\bar{F}^{\prime \prime}\left(s_{i}\right), \bar{F}^{\prime \prime}\left(s_{i+1}\right)\right)\right\} .
\end{aligned}
$$

By summing over $i$ from 0 to $n-1$ and taking into account that $\left|\bar{F}^{\prime \prime}\right|$ is $\varphi$-convex to get

$$
\begin{aligned}
& \left|S(\bar{F}, \mathscr{P})-\int_{\xi_{1}}^{\xi_{2}} \bar{F}(s) \mathrm{d} s\right| \\
& \quad \leq \sum_{i=0}^{n-1} \frac{\left(s_{i+1}-s_{i}\right)^{3}}{81}\left[\bar{F}^{\prime \prime}\left(s_{i}\right)+\frac{1}{2} \varphi\left(\bar{F}^{\prime \prime}\left(s_{i}\right), \bar{F}^{\prime \prime}\left(s_{i+1}\right)\right)\right],
\end{aligned}
$$

which completes our proof.

Corollary 6. Theorem 5 with $\varphi(x, y)=y-x$ becomes

$$
\left|E_{s}(\bar{F}, \mathscr{P})\right| \leq \frac{1}{162} \sum_{i=0}^{n-1}\left(s_{i+1}-s_{i}\right)^{3}\left[\bar{F}^{\prime \prime}\left(s_{i}\right)+\bar{F}^{\prime \prime}\left(s_{i+1}\right)\right] .
$$

Theorem 6. Let $\bar{F}: \mathfrak{I} \subseteq[0, \infty) \longrightarrow R$ be a twice differentiable function on $\mathfrak{\Im}$ such that $\bar{F}^{\prime \prime} \in L_{1}\left[\xi_{1}, \xi_{2}\right]$, where $\xi_{1}, \xi_{2} \in \mathfrak{J}$ with $\xi_{1}<\xi_{2}$. If $\left|\bar{F}^{\prime \prime}\right|$ is $\varphi$-quasiconvex on $\left[\xi_{1}, \xi_{2}\right]$, then for every division $\mathscr{P}$ of $\left[\xi_{1}, \xi_{2}\right]$ we have

$$
\left|S(\bar{F}, \mathscr{P})-\int_{\xi_{1}}^{\xi_{2}} \bar{F}(s) \mathrm{d} s\right| \leq \frac{1}{81} \sum_{i=0}^{n-1}\left(s_{i+1}-s_{i}\right)^{3} \max \left\{\left|\bar{F}^{\prime \prime}\left(s_{i}\right)\right|,\left|\bar{F}^{\prime \prime}\left(s_{i}\right)\right|+\varphi\left(\left|\bar{F}^{\prime \prime}\left(s_{i}\right)\right|,\left|\bar{F}^{\prime \prime}\left(s_{i+1}\right)\right|\right)\right\} .
$$

Proof. By applying Theorem 3 and by the same method used for proof of the previous theorem, we can produce the desired result.

Proposition 5. Let $\bar{F}: \mathfrak{I} \subseteq[0, \infty) \longrightarrow R$ be a twice differentiable function on $\mathfrak{\Im}$ such that $\bar{F}^{\prime \prime} \in L_{1}\left[\xi_{1}, \xi_{2}\right]$, where $\xi_{1}, \xi_{2} \in \mathfrak{\Im}$ with $\xi_{1}<\xi_{2}$. If $\left|\bar{F}^{\prime \prime}\right|^{q} q \geq 1$ is $\varphi$-convex on $\left[\xi_{1}, \xi_{2}\right]$, then we have

$$
\left|E_{s}(\bar{F}, \mathscr{P})\right| \leq\left(\frac{1}{162}\right)^{1-(1 / q)} \sum_{i=0}^{n-1}\left(s_{i+1}-s_{i}\right)^{3}\left[K_{\varphi}^{q}\left(\bar{F}^{\prime \prime}\left(s_{i}\right), \bar{F}^{\prime \prime}\left(s_{i+1}\right)\right)\right],
$$


where

$$
\begin{aligned}
K_{\varphi}^{q}\left(\bar{F}^{\prime \prime}\left(s_{i}\right), \bar{F}^{\prime \prime}\left(s_{i+1}\right)\right)= & \left(\frac{1}{162}\left|\bar{F}^{\prime \prime}\left(s_{i}\right)\right|^{q}+\frac{59}{31104} \varphi\left(\left|\bar{F}^{\prime \prime}\left(s_{i}\right)\right|^{q},\left|\bar{F}^{\prime \prime}\left(s_{i+1}\right)\right|^{q}\right)\right)^{1 / q} \\
& +\left(\frac{1}{162}\left|\bar{F}^{\prime \prime}\left(s_{i}\right)\right|^{q}+\frac{133}{31104} \varphi\left(\left|\bar{F}^{\prime \prime}\left(s_{i}\right)\right|^{q},\left|\bar{F}^{\prime \prime}\left(s_{i+1}\right)\right|^{q}\right)\right)^{1 / q} .
\end{aligned}
$$

Proof. The proof follows from Theorem 2 directly.

$\xi_{1}, \xi_{2} \in \mathfrak{J}$ with $\xi_{1}<\xi_{2}$. If $\left|\bar{F}^{\prime \prime}\right|^{q} q \geq 1$ is $\varphi$-quasiconvex on

Proposition 6. Let $\bar{F}: \mathfrak{J} \subseteq[0, \infty) \longrightarrow R$ be a twice differ-

entiable function on $\mathfrak{J}$ such that $\bar{F}^{\prime \prime} \in L_{1}\left[\xi_{1}, \xi_{2}\right]$, where

$$
\left|E_{s}(\bar{F}, \mathscr{P})\right| \leq \frac{1}{81} \sum_{i=0}^{n-1}\left(s_{i+1}-s_{i}\right)^{3} \max \left\{\left|\bar{F}^{\prime \prime}\left(s_{i}\right)\right|^{q},\left|\bar{F}^{\prime \prime}\left(s_{i}\right)\right|^{q}+\varphi\left(\left|\bar{F}^{\prime \prime}\left(s_{i+1}\right)\right|^{q},\left|\bar{F}^{\prime \prime}\left(s_{i}\right)\right|^{q}\right)\right\} .
$$

Proof. The proof follows from Theorem 4 directly.

4.3. Applications to the Midpoint Formula. Let $\mathscr{P}$ be a partition as before. Here we consider the midpoint formula:

$$
M(\bar{F}, \mathscr{P})=\sum_{i=0}^{n-1}\left(s_{i+1}-s_{i}\right) \bar{F}\left(\frac{s_{i}+s_{i+1}}{2}\right) .
$$

Suppose that the function $\bar{F}:\left[\xi_{1}, \xi_{2}\right] \longrightarrow R$ is differentiable with $\bar{F}^{\prime \prime}(x)$ existing on $\left(\xi_{1}, \xi_{2}\right)$ and $K=\sup _{x \in\left(\xi_{1}, \xi_{2}\right)}\left|\bar{F}^{\prime \prime}(x)\right|<\infty$, and then, we have

$$
I=\int_{\xi_{1}}^{\xi_{2}} \bar{F}(s) \mathrm{d} s=M(\bar{F}, \mathscr{P})+E_{M}(\bar{F}, \mathscr{P}),
$$

where the approximation error $E_{M}(\bar{F}, \mathscr{P})$ satisfies

$$
\left|E_{M}(\bar{F}, \mathscr{P})\right| \leq \frac{K}{24} \sum_{i=0}^{n-1}\left(s_{i+1}-s_{i}\right)^{3} .
$$

Proposition 7. Let $\bar{F}: \mathfrak{I} \longrightarrow R$ be a twice differentiable function on $\mathfrak{J}, \xi_{1}, \xi_{2} \in \mathfrak{J}$ with $\xi_{1}<\xi_{2}$. If $\left|\bar{F}^{\prime \prime}\right|$ is $\varphi$-convex on $\left[\xi_{1}, \xi_{2}\right]$, then for any division $\mathscr{P}$ of $\left[\xi_{1}, \xi_{2}\right]$, we have

$$
\begin{aligned}
& \left|E_{M}(\bar{F}, \mathscr{P})\right| \\
& \quad \leq \frac{1}{81} \sum_{i=0}^{n-1}\left(s_{i+1}-s_{i}\right)^{3}\left[\left|\bar{F}^{\prime \prime}\left(s_{i}\right)\right|+\frac{1}{2} \varphi\left(\left|\bar{F}^{\prime \prime}\left(s_{i}\right)\right|,\left|\bar{F}^{\prime \prime}\left(s_{i+1}\right)\right|\right)\right] .
\end{aligned}
$$

Proof. By applying Corollary 1 on the subintervals $\left[s_{i}, s_{i+1}\right],(i=0,1, \ldots, n-1)$ of the division $\mathscr{P}$, to get

$$
\begin{aligned}
& \left|\left(s_{i+1}-s_{i}\right) \bar{F}\left(\frac{s_{i+1}+s_{i}}{2}\right)-\int_{s_{i}}^{s_{i+1}} \bar{F}(s) \mathrm{d} s\right| \\
& \quad \leq \frac{\left(s_{i+1}-s_{i}\right)^{3}}{81}\left[\left|\bar{F}^{\prime \prime}\left(s_{i}\right)\right|+\frac{1}{2} \varphi\left(\left|\bar{F}^{\prime \prime}\left(s_{i}\right)\right|,\left|\bar{F}^{\prime \prime}\left(s_{i+1}\right)\right|\right)\right] .
\end{aligned}
$$

By summing over $i$ from 0 to $n-1$ to get

$$
\begin{aligned}
& \left|E_{M}(\bar{F}, \mathscr{P})\right| \\
& \quad \leq \frac{1}{81} \sum_{i=0}^{n-1}\left(s_{i+1}-s_{i}\right)^{3}\left[\left|\bar{F}^{\prime \prime}\left(s_{i}\right)\right|+\frac{1}{2} \varphi\left(\left|\bar{F}^{\prime \prime}\left(s_{i}\right)\right|,\left|\bar{F}^{\prime \prime}\left(s_{i+1}\right)\right|\right)\right],
\end{aligned}
$$

which completes our proof.

Proposition 8. Let $\bar{F}: \mathfrak{I} \longrightarrow R$ be a twice differentiable function on $\mathfrak{J}, \xi_{1}, \xi_{2} \in \mathfrak{I}$ with $\xi_{1}<\xi_{2}$. If $\left|\bar{F}^{\prime \prime}\right|^{q}$ is $\varphi$-convex on $\left[\xi_{1}, \xi_{2}\right]$ and $q \geq 1$, then for any division $\mathscr{P}$ of $\left[\xi_{1}, \xi_{2}\right]$, we have

$$
\begin{aligned}
& \left|E_{M}(\bar{F}, \mathscr{P})\right| \\
& \quad \leq\left(\frac{1}{162}\right)^{1-(1 / q)} \sum_{i=0}^{n-1}\left(s_{i+1}-s_{i}\right)^{3}\left[K_{\varphi}^{q}\left(\bar{F}^{\prime \prime}\left(s_{i}\right), \bar{F}^{\prime \prime}\left(s_{i+1}\right)\right)\right],
\end{aligned}
$$

where

$$
\begin{aligned}
K_{\varphi}^{q}\left(\bar{F}^{\prime \prime}\left(s_{i}\right), \bar{F}^{\prime \prime}\left(s_{i+1}\right)\right) \\
=\left(\frac{1}{162}\left|\bar{F}^{\prime \prime}\left(s_{i}\right)\right|^{q}+\frac{59}{31104} \varphi\left(\left|\bar{F}^{\prime \prime}\left(s_{i}\right)\right|^{q},\left|\bar{F}^{\prime \prime}\left(s_{i+1}\right)\right|^{q}\right)\right)^{1 / q} \\
\quad+\left(\frac{1}{162}\left|\bar{F}^{\prime \prime}\left(s_{i}\right)\right|^{q}+\frac{133}{31104} \varphi\left(\left|\bar{F}^{\prime \prime}\left(s_{i}\right)\right|^{q},\left|\bar{F}^{\prime \prime}\left(s_{i+1}\right)\right|^{q}\right)\right)^{1 / q} .
\end{aligned}
$$


Proof. By applying Corollary 2 on the subintervals $\left[s_{i}, s_{i+1}\right],(i=0,1, \ldots, n-1)$ of the division $\mathscr{P}$ to get

$$
\begin{aligned}
& \left|\left(s_{i+1}-s_{i}\right) \bar{F}\left(\frac{s_{i+1}+s_{i}}{2}\right)-\int_{s_{i}}^{s_{i+1}} \bar{F}(s) \mathrm{d} s\right| \\
& \leq\left(\frac{1}{162}\right)^{1-(1 / q)}\left(s_{i+1}-s_{i}\right)^{3}\left[K_{\varphi}^{q}\left(\bar{F}^{\prime \prime}\left(s_{i}\right), \bar{F}^{\prime \prime}\left(s_{i+1}\right)\right)\right],
\end{aligned}
$$

where

$$
\begin{aligned}
K_{\varphi}^{q}\left(\bar{F}^{\prime \prime}\left(s_{i}\right), \bar{F}^{\prime \prime}\left(s_{i+1}\right)\right) \\
=\left(\frac{1}{162}\left|\bar{F}^{\prime \prime}\left(s_{i}\right)\right|^{q}+\frac{59}{31104} \varphi\left(\left|\bar{F}^{\prime \prime}\left(s_{i}\right)\right|^{q},\left|\bar{F}^{\prime \prime}\left(s_{i+1}\right)\right|^{q}\right)\right)^{1 / q} \\
\quad+\left(\frac{1}{162}\left|\bar{F}^{\prime \prime}\left(s_{i}\right)\right|^{q}+\frac{133}{31104} \varphi\left(\left|\bar{F}^{\prime \prime}\left(s_{i}\right)\right|^{q},\left|\bar{F}^{\prime \prime}\left(s_{i+1}\right)\right|^{q}\right)\right)^{1 / q} .
\end{aligned}
$$

By summing over $i$ from 0 to $n-1$ to get $\left|E_{M}(\bar{F}, \mathscr{P})\right|$

$$
\leq\left(\frac{1}{162}\right)^{1-(1 / q)} \sum_{i=0}^{n-1}\left(s_{i+1}-s_{i}\right)^{3}\left[K_{\varphi}^{q}\left(\bar{F}^{\prime \prime}\left(s_{i}\right), \bar{F}^{\prime \prime}\left(s_{i+1}\right)\right)\right]
$$

which completes our proof.

Proposition 9. Let $\bar{F}: \mathfrak{I} \longrightarrow R$ be a twice differentiable function on $\mathfrak{J}, \xi_{1}, \xi_{2} \in \mathfrak{J}$ with $\xi_{1}<\xi_{2}$. If $\left|\bar{F}^{\prime \prime}\right|$ is $\varphi$-quasiconvex on $\left[\xi_{1}, \xi_{2}\right]$, then for any division $\mathscr{P}$ of $\left[\xi_{1}, \xi_{2}\right]$, we have

$$
\left|E_{M}(\bar{F}, \mathscr{P})\right| \leq \frac{1}{81} \sum_{i=0}^{n-1}\left(s_{i+1}-s_{i}\right)^{3} \max \left\{\left|\bar{F}^{\prime \prime}\left(s_{i}\right)\right|,\left|\bar{F}^{\prime \prime}\left(s_{i}\right)\right|+\varphi\left(\left|\bar{F}^{\prime \prime}\left(s_{i}\right)\right|,\left|\bar{F}^{\prime \prime}\left(s_{i+1}\right)\right|\right)\right\} .
$$

Proof. By applying Corollary 3 on the subintervals $\left[s_{i}, s_{i+1}\right],(i=0,1, \ldots, n-1)$ of the division $\mathscr{P}$ to get

By summing over $i$ from 0 to $n-1$ to get

$$
\begin{aligned}
& \left|\left(s_{i+1}-s_{i}\right) \bar{F}\left(\frac{s_{i+1}+s_{i}}{2}\right)-\int_{s_{i}}^{s_{i+1}} \bar{F}(s) \mathrm{d} s\right| \\
& \quad \leq \frac{\left(s_{i+1}-s_{i}\right)^{3}}{81} \max \left\{\left|\bar{F}^{\prime \prime}\left(s_{i}\right)\right|,\left|\bar{F}^{\prime \prime}\left(s_{i}\right)\right|+\varphi\left(\left|\bar{F}^{\prime \prime}\left(s_{i}\right)\right|,\left|\bar{F}^{\prime \prime}\left(s_{i+1}\right)\right|\right)\right\} .
\end{aligned}
$$

$$
\left|E_{M}(\bar{F}, \mathscr{P})\right| \leq \frac{1}{81} \sum_{i=0}^{n-1}\left(s_{i+1}-s_{i}\right)^{3} \max \left\{\left|\bar{F}^{\prime \prime}\left(s_{i}\right)\right|,\left|\bar{F}^{\prime \prime}\left(s_{i}\right)\right|+\varphi\left(\left|\bar{F}^{\prime \prime}\left(s_{i}\right)\right|,\left|\bar{F}^{\prime \prime}\left(s_{i+1}\right)\right|\right)\right\}
$$

which completes our proof.

Proposition 10. Let $\bar{F}: \mathfrak{S} \longrightarrow R$ be a twice differentiable function on $\mathfrak{J}, \xi_{1}, \xi_{2} \in \mathfrak{J}$ with $\xi_{1}<\xi_{2}$. If $\left|\bar{F}^{\prime \prime}\right|^{q}$ is $\varphi$-quasiconvex on $\left[\xi_{1}, \xi_{2}\right]$ and $q \geq 1$, then in (30), for every division $\mathscr{P}$ of $\left[\xi_{1}, \xi_{2}\right]$, we have

$$
\left|E_{M}(\bar{F}, \mathscr{P})\right| \leq \frac{1}{81} \sum_{i=0}^{n-1}\left(s_{i+1}-s_{i}\right)^{3} \max \left\{\left|\bar{F}^{\prime \prime}\left(s_{i}\right)\right|^{q},\left|\bar{F}^{\prime \prime}\left(s_{i}\right)\right|^{q}+\varphi\left(\left|\bar{F}^{\prime \prime}\left(s_{i+1}\right)\right|^{q},\left|\bar{F}^{\prime \prime}\left(s_{i}\right)\right|^{q}\right)\right\} .
$$

Proof. By applying Corollary 4 on the subintervals $\left[s_{i}, s_{i+1}\right],(i=0,1, \ldots, n-1)$ of the division $\mathscr{P}$, we get

$$
\begin{aligned}
& \left|\left(s_{i+1}-s_{i}\right) \bar{F}\left(\frac{s_{i+1}+s_{i}}{2}\right)-\int_{s_{i}}^{s_{i+1}} \bar{F}(s) \mathrm{d} s\right| \\
& \quad \leq \frac{\left(s_{i+1}-s_{i}\right)^{3}}{81} \max \left\{\left|\bar{F}^{\prime \prime}\left(s_{i}\right)\right|^{q},\left|\bar{F}^{\prime \prime}\left(s_{i}\right)\right|^{q}+\varphi\left(\left|\bar{F}^{\prime \prime}\left(s_{i+1}\right)\right|^{q},\left|\bar{F}^{\prime \prime}\left(s_{i}\right)\right|^{q}\right)\right\} .
\end{aligned}
$$




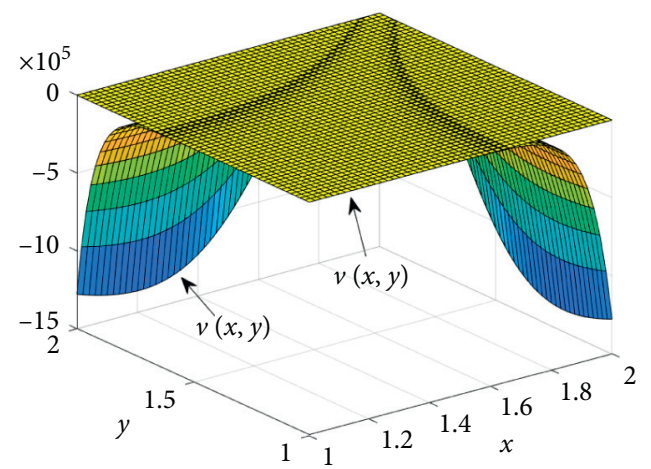

(a)

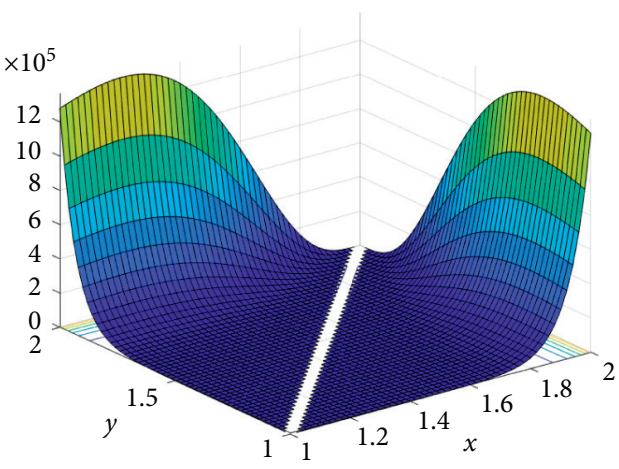

(b)

Figure 1: Plot illustrations for inequality (42). (a) For $v(x ; y)$ and $V(x ; y)$. (b) For $V(x ; y)-v(x ; y)$.

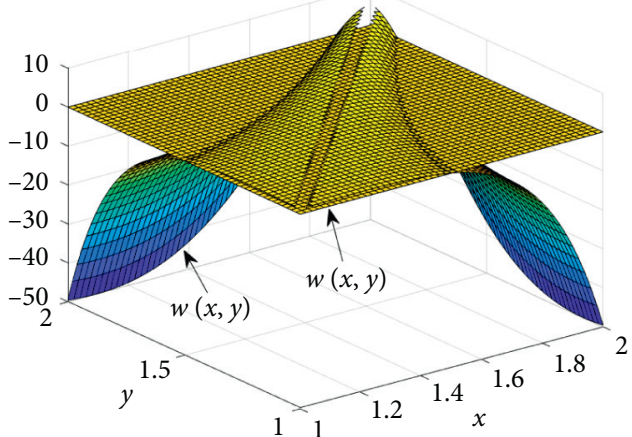

(a)

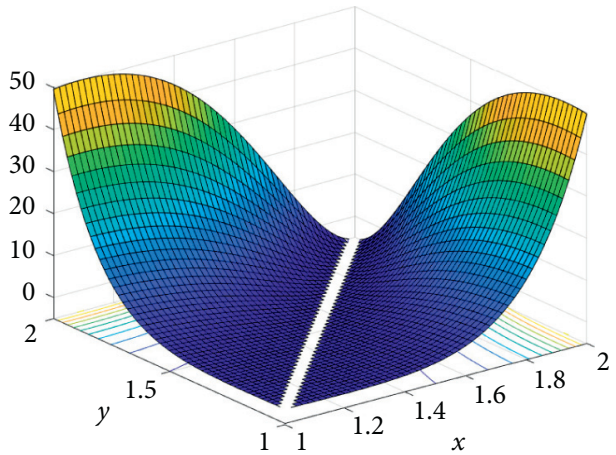

(b)

Figure 2: Plot illustrations for inequality (44). (a) For $w(x ; y)$ and $W(x ; y)$. (b) For $W(x, y)-w(x, y)$.

By summing over $i$ from 0 to $n-1$ to get

$$
\left|E_{M}(\bar{F}, \mathscr{P})\right| \leq \frac{1}{81} \sum_{i=0}^{n-1}\left(s_{i+1}-s_{i}\right)^{3} \max \left\{\left|\bar{F}^{\prime \prime}\left(s_{i}\right)\right|^{q},\left|\bar{F}^{\prime \prime}\left(s_{i}\right)\right|^{q}+\varphi\left(\left|\bar{F}^{\prime \prime}\left(s_{i+1}\right)\right|^{q},\left|\bar{F}^{\prime \prime}\left(s_{i}\right)\right|^{q}\right)\right\}
$$

which rearranges to the proof.

\section{Illustrative Plots}

Finally, we present two three-dimensional plots to demonstrate the validity of the inequalities (42) and (44) in the case of $\varphi$-convex and $\varphi$-quasiconvex functions, respectively.

From inequality (42), we can define

$$
\begin{aligned}
v(x, y) & :=q \frac{1}{3} \mathscr{A}\left(x^{4}, y^{4}\right)+\frac{2}{3} \mathscr{A}^{4}(x, y)-\mathscr{L}_{5}^{5}(x, y) \\
V(x, y) & :=q \frac{(y-x)^{2}}{27}\left[8 x^{2}+y^{2}\right] .
\end{aligned}
$$

Thus, Figure 1 represents the plot of inequality (42) and $V(x, y)-v(x, y)$.

From inequality (44), we can define

$$
\begin{aligned}
w(x, y) & =\frac{1}{3} \mathscr{A}\left(x^{2}, y^{2}\right)+\frac{2}{3} \mathscr{A}^{2}(x, y)-\mathscr{L}_{3}^{3}(x, y) \\
W(x, y) & =\frac{2(y-x)^{2}}{81} .
\end{aligned}
$$

Thus, Figure 2 represents the plot of inequality (44) and $W(x, y)-w(x, y)$.

\section{Conclusion}

In this study, we have considered Simpson's type integral inequalities for the $\varphi$-convex and $\varphi$-quasiconvex functions in the second derivative sense. Some special cases of our findings are investigated to show the powerfulness of our results. Also, the proposed inequalities can be applied to other mathematical and statistical models, as we have shown in Section 4. 


\section{Data Availability}

No data were used to support this study.

\section{Conflicts of Interest}

The authors declare that they have no conflicts of interest.

\section{Acknowledgments}

The authors want to give thanks to the Dirección de investigación from Pontificia Universidad Católica del Ecuador for technical support to our research project entitled: "Algunas desigualdades integrales para funciones convexas generalizadas y aplicaciones". The second author would like to thank Prince Sultan University for funding this work through research group Nonlinear Analysis Methods in Applied Mathematics (NAMAM) group number RGDES-2017-01-17.

\section{References}

[1] V. Lakshmikantham and S. Leela, Differential and Integral Inequalities: Theory and Applications: Volume I: Ordinary Differential Equations, Academic Press, New York, NY, USA, 1969.

[2] W. Walter, Differential and Integral Inequalities, Vol. 55, Springer Science \& Business Media, Berlin, Germany, 2012.

[3] P. O. Mohammed, "New integral inequalities for preinvex functions via generalized beta function," Journal of Interdisciplinary Mathematics, vol. 22, no. 4, pp. 539-549, 2019.

[4] P. O. Mohammed, "Some new Hermite-Hadamard type inequalities for MT-convex functions on differentiable coordinates," Journal of King Saud University-Science, vol. 30, no. 2, pp. 258-262, 2018.

[5] T. Abdeljawad, "A Lyapunov type inequality for fractional operators with nonsingular Mittag-Leffler kernel," Journal of Inequalities and Applications, vol. 2017, no. 1, p. 130, 2017.

[6] A. Fernandez and P. Mohammed, "Hermite-Hadamard inequalities in fractional calculus defined using Mittag-Leffler kernels," Mathematical Methods in the Applied Sciences, pp. 1-18, 2020.

[7] P. O. Mohammed, "Some integral inequalities of fractional quantum type," Malaya Journal of Matematik, vol. 4, no. 1, pp. 93-99, 2016.

[8] D. Baleanu, P. O. Mohammed, and S. Zeng, "Inequalities of trapezoidal type involving generalized fractional integrals," Alexandria Engineering Journal, 2020.

[9] P. O. Mohammed, "Inequalities of $(k, s),(k, h)$-type for Riemann-liouville fractional integrals," Applied Mathematics E-Notes, vol. 17, pp. 199-206, 2017.

[10] P. O. Mohammed, "Hermite-Hadamard inequalities for Riemann-Liouville fractional integrals of a convex function with respect to a monotone function," Mathematical Methods in the Applied Sciences, pp. 1-11, 2019.

[11] P. O. Mohammed, "On new trapezoid type inequalities for," TJANT, vol. 6, no. 4, pp. 125-128, 2018.

[12] P. O. Mohammed and T. Abdeljawad, "Modification of certain fractional integral inequalities for convex functions," Advances in Difference Equations, vol. 2020, no. 1, p. 69, 2020.

[13] P. O. Mohammed and I. Brevik, "A new version of the hermite-hadamard inequality for Riemann-liouville fractional integrals," Symmetry, vol. 12, no. 4, p. 610, 2020.
[14] P. O. Mohammed and M. Z. Sarikaya, "Hermite-Hadamard type inequalities for F-convex function involving fractional integrals," Journal of Inequalities and Applications, vol. 2018, no. 1 , p. 359, 2018.

[15] P. O. Mohammed and M. Z. Sarikaya, "On generalized fractional integral inequalities for twice differentiable convex functions," Journal of Computational and Applied Mathematics, vol. 372, p. 112740, 2020.

[16] P. O. Mohammed, M. Z. Sarikaya, and D. Baleanu, "On the generalized hermite-hadamard inequalities via the tempered fractional integrals," Symmetry, vol. 12, no. 4, p. 595, 2020.

[17] F. Qi, P. O. Mohammed, J.-C. Yao, and Y.-H. Yao, "Generalized fractional integral inequalities of Hermite-Hadamard type for $\alpha$-convex functions," Journal of Inequalities and Applications, vol. 2019, no. 1, p. 135, 2019.

[18] M. Alomari, M. Darus, and S. Dragomir, "New inequalities of Hermite-Hadamard type for functions whose second derivatives absolute values are quasi-convex," RGMIA Research Report Collection, vol. 2, no. 3, pp. 1-14, 2009.

[19] M. Alomari, M. Darus, and S. Dragomir, "New inequalities of simpson's type for s-convex functions with applications," RGMIA Research Report Collection, vol. 4, no. 12, pp. 1-18, 2009.

[20] S. Dragomir, R. Agarwal, and P. Cerone, “On simpson's inequality and applications," Journal of Inequalities and Applications, vol. 5, pp. 533-579, 2000.

[21] Z. Liu, "An inequality of Simpson type," Proceedings of the Royal Society A: Mathematical, Physical and Engineering Sciences, vol. 461, no. 2059, pp. 2155-2158, 2005.

[22] Y. C. Rangel and M. J. Vivas, "Ostrowski type inequalities for functions whose second derivative are convex generalized," Applied Mathematics \& Information Sciences, vol. 12, no. 6, pp. 1055-1064, 2018.

[23] M. Z. Sarikaya, E. Set, and M. E. Ozdemir, "On new inequalities of simpson's type for functions whose second derivatives absolute values are convex," Journal of Applied Mathematics, Statistics and Informatics, vol. 9, no. 1, pp. 37-45, 2013.

[24] M. J. Vivas, "Fèjer type inequalities for $(s, m)$-convex functions in second sense," Applied Mathematics \& Information Sciences, vol. 10, no. 5, pp. 1689-1696, 2016.

[25] M. J. Vivas and C. García, "Ostrowski type inequalities for functions whose derivatives are (m, h1, h2)-convex," Applied Mathematics \& Information Sciences, vol. 11, no. 1, pp. 79-86, 2019.

[26] J. M. Viloria and M. V. Cortez, "Hermite-hadamard type inequalities for harmonically convex functions on $n$-coordinates," Applied Mathematics \& Information Sciences Letters, vol. 6, no. 2, pp. 53-58, 2018.

[27] M. V. Cortez, "Relative strongly h-convex functions and integral inequalities," Applied Mathematics \& Information Sciences Letters, vol. 4, no. 2, pp. 39-45, 2016.

[28] M. Bracamonte, J. Gimènez, and M. Vivas-Cortez, "Hermitehadamard-fejer type inequalities for strongly (s,m)-convex functions with modulus c, in second sense," Applied Mathematics \& Information Sciences, vol. 10, no. 6, pp. 2045-2053, 2016.

[29] M. J. Vivas, J. Hernández, and N. Merentes, "New hermitehadamard and jensen type inequalities for h-convex functions on fractal sets," Rev. Colombiana Mat, vol. 50, no. 2, pp. 145-164, 2016.

[30] F. Ertuğral and M. Z. Sarikaya, "Simpson type integral inequalities for generalized fractional integral," RACSAM, vol. 113, pp. 3115-3124, 2019. 
[31] E. Set, A. Akdemir, and E. Özdemir, "Simpson type integral inequalities for convex functions via Riemann-liouville integrals," Filomat, vol. 31, no. 14, pp. 4415-4420, 2017.

[32] S. Kermausuor, "Simpson's type inequalities for $\eta$-convex functions via $k$-Riemann-Liouville fractional integrals," ACUTM, vol. 23, pp. 1-8, 2019.

[33] M. E. Gordji, M. R. Delavar, and M. D. L. Sen, "On $\varphi$-convex functions," Journal of Mathematical Inequalities, vol. 10, no. 1, pp. 173-183, 2016.

[34] E. R. Nwaeze and D. F. M. Torres, "New inequalities for $\varphi$-quasiconvex functions," in Frontiers in Functional Equations and Analytic Inequalities (FEAI), G. Anastassiou and J. Rassias, Eds., Springer, New York, NY, USA, 2019. 